\title{
The rational parts of one-loop QCD amplitudes III: The six-gluon case
}

\author{
Zhi-Guang Xiao*1,2, Gang Yang ${ }^{\dagger 2}$ and Chuan-Jie Zhu ${ }^{\ddagger 2,3}$
}

November 13, 2018

\author{
${ }^{1}$ The Interdisciplinary Center of Theoretical Studies, Chinese \\ Academy of Sciences, P. O. Box 2735, Beijing 100080, P. R. China \\ ${ }^{2}$ Institute of Theoretical Physics, Chinese Academy of Sciences \\ P. O. Box 2735, Beijing 100080, P. R. China \\ ${ }^{3}$ Center of Mathematical Science, Zhejiang University \\ Hangzhou 310027, P. R. China
}

\begin{abstract}
The rational parts of 6-gluon one-loop amplitudes with scalars circulating in the loop are computed by using the newly developed method for computing the rational parts directly from Feynman integrals. We present the analytic results for the two MHV helicity configurations: $\left(1^{-} 2^{+} 3^{+} 4^{-} 5^{+} 6^{+}\right)$and $\left(1^{-} 2^{+} 3^{-} 4^{+} 5^{+} 6^{+}\right)$, and the two NMHV helicity configurations: $\left(1^{-} 2^{-} 3^{+} 4^{-} 5^{+} 6^{+}\right)$and $\left(1^{-} 2^{+} 3^{-} 4^{+} 5^{-} 6^{+}\right)$. Combined with the previously computed results for the cut-constructible part, our results are the last missing pieces for the complete partial helicity amplitudes of the 6-gluon one-loop QCD amplitude.
\end{abstract}

\footnotetext{
*E-mail: zhgxiao@itp.ac.cn

${ }^{\dagger}$ E-mail: yangg@itp.ac.cn

$\ddagger$ E-mail: zhucj@itp.ac.cn
} 


\section{Introduction}

In two previous papers $[1,2]$, we developed a method of computing the rational parts of one-loop amplitudes directly from Feynman integrals and applied it to compute the rational parts of the 5-gluon amplitudes. The result agrees with the well-known result of Bern, Dixon and Kosower [3] obtained firstly by using string-inspired methods. In this paper we use our method to compute explicitly the rational parts of the 6-gluon amplitudes in QCD. For all the 8 independent helicity configurations, we computed explicitly all cases except the all plus helicity case, as this is a well-known finite amplitude [4]. For the three helicity configurations which have explicit results published $[5,6,7,8]$, we found complete analytic agreement. For the remaining two MHV cases we compared with the results of Berger, Bern, Dixon, Forde and Kosower [9] obtained by using the bootstrap recursive relations. We find complete agreement. The results obtained by Berger, Bern, Dixon, Forde and Kosower in [9] are more general all-multiplicity one-loop MHV amplitudes. Our method shows its power by firstly obtaining also the analytic results for the rational parts of the remaining 2 non-MHV helicity configurations of the one-loop 6-gluon amplitude. Our method and the bootstrap recursive approach complement each other quite nicely.

The constant effort to calculate higher-point one-loop amplitudes in general and 6-point amplitude in particular lies in the application to the forthcoming experimental program at CERN's Large Hadron Collider (LHC), as there are lots of processes with many particles as final states [10]. We refer the reader to [1] for a discussion and extensive references for the recent efforts in computing the multi-particle one-loop amplitudes and the recent developments inspired by twistor string theory $[11,12]$. Here we will concentrate on the 6-gluon one-loop amplitude in QCD.

In principle any one-loop amplitude in QCD can be computed by using Feynman diagrams and Feynman rules. The 4-parton amplitude was computed in this way by computing the numerous Feynman diagrams [13]. However using this brute-force method to compute even the 5-gluon one-loop amplitude was quite a challenge. Starting from 1987, Bern and Kosower developed the method to compute one-loop amplitudes by string theory [14]. The 5-gluon one-loop amplitude was computed firstly in this way by Bern,

Dixon and Kosower [3]. It turns out that the lessons learnt from string theory are quite useful. In fact one can forget about string theory and only keeps the 
tricks learnt in these lessons $[16,17,18]$. Subsequently other 5-parton amplitudes were later computed by using either standard Feynman diagrammatic technique [19] or supersymmetric decomposition and perturbative unitarity [20].

However the string theory (inspired) method is still not powerful enough and the analytic computation of the 6-gluon one-loop amplitude is quite a challenge. Only for special helicity configurations and special models, some analytic results are known $[4,5,21]$.

To make progress, it is a good strategy to decompose the QCD amplitude into simpler ones by using the supersymmetric decomposition:

$$
A^{Q C D}=A^{N=4}-4 A^{N=1 \text { chiral }}+A^{N=0 \text { or scalar },}
$$

where $A^{Q C D}$ denotes an amplitude with only a gluon circulating in the loop, $A^{N=4,1}$ have the full $N=4,1$ multiplets circulating in the loop, and $A^{N=0}$ has only a complex scalar in the loop.

By using the general properties of the one-loop amplitude, Bern, Dunbar, Dixon and Kosower proved that the supersymmetric amplitude $A^{N=4,1}$ are completely determined by 4 -dimensional unitarity [15], i.e. the amplitude is completely cut-constructible and the rational part is vanishing (see [1] for more detail explanation). For MHV helicity configurations, explicit results were obtained for $A^{N=4}$ in [15]. The recent development of using MHV vertices to compute one-loop amplitudes leads to many new results for the cut-constructible part $[22,23,24,25,26,27,28,29,30,31,32]$. In particular, Bedford, Brandhuber, Spence and Travaglini $[22,26]$ applied the MHV vertices to one-loop calculations. Britto, Buchbinder, Cachazo, Feng and Mastrolia [31, 32, 33] developed an efficient technique for evaluating the rational coefficients in an expansion of the one-loop amplitude in terms of scalar box, triangle and bubble integrals (the cut-constructible part, see [1] for details). By using their technique, it is much easier to calculate the coefficients of box integrals without doing any integration. Recently, Britto, Feng and Mastrolia completed the computation of the cut-constructible terms for all the 6-gluon helicity amplitudes [33].

In order to complete the QCD calculation for the 6-gluon helicity amplitudes, the remaining challenge is to compute the rational parts of the helicity amplitudes with scalars circulating in the loop.

As we reviewed in [1], there are various approaches $[34,35,36,37,6,7]$ to compute the rational parts. In particular, Bern, Dixon and Kosower $[6,7]$ 
developed the bootstrap recursive approach which has lead to quite general results $[38,8,9]$. In this paper we will use the approach as developed in [1] and apply it to compute the rational parts of the 6-gluon one-loop amplitudes in QCD (see also [9]) which are the last missing pieces for the complete partial helicity amplitudes of the 6-gluon one-loop QCD amplitude. The usefulness of this approach was checked in [2] by reproducing (the rational parts of) the well-known 5-gluon one-loop amplitudes of Bern, Dixon and Kosower [3]. See [16] for a general review.

For the helicity configurations $\left(1^{+} 2^{+} 3^{+} 4^{+} 5^{+} 6^{+}\right)$and $\left(1^{-} 2^{+} 3^{+} 4^{+} 5^{+} 6^{+}\right)$, the cut-constructible parts are zero and the rational parts were known already $[4,5]$. These rational parts have also been derived by using the bootstrap recursive method [8]. We also computed the rational part of $\left(1^{-} 2^{+} 3^{+} 4^{+} 5^{+} 6^{+}\right)$ by using our new method and found the same analytic result.

For the so call "split-helicity" configurations $\left(1^{-} 2^{-} 3^{+} 4^{+} 5^{+} 6^{+}\right)$and $\left(1^{-} 2^{-} 3^{-}\right.$ $\left.4^{+} 5^{+} 6^{+}\right)$, the computation of the rational parts is a recent achievement $[7,8]$. By using our method, we also computed the rational parts for these two helicity configurations. The results obtained agree with their results. The proof is done by using Mathematica by expressing all spinor products in terms of 12 independent spinor products after explicitly solving the equations from momenta conservation. We will not give any details in this paper.

A general strategy for computing the remaining 4 helicity configurations was outlined in [6]. Explicit results for the all-multiplicity MHV one-loop amplitudes, including the remaining two MHV helicity configurations of the 6 gluons $\left(1^{-} 2^{+} 3^{-} 4^{+} 5^{+} 6^{+}\right)$and $\left(1^{-} 2^{+} 3^{+} 4^{-} 5^{+} 6^{+}\right)$, were given in [9]. Independently we also obtained the explicit results of the rational parts for these two MHV helicity configurations of the 6 gluons, see Sects. 4 and 5 .

Due to the appearance of the 3-mass triangle integrals in the remaining 2 non-MHV helicity configurations $\left(1^{-} 2^{-} 3^{+} 4^{-} 5^{+} 6^{+}\right)$and $\left(1^{-} 2^{+} 3^{-} 4^{+} 5^{-} 6^{+}\right)$, the analytic results are much more complicated. The results can be actually presented in a compact form by exploiting the symmetry of the amplitude. As we showed in [1], the 2-mass-hard box rational parts can be expressed in terms of 2-mass and 3-mass triangle integrals. If we write the final results in terms of the 2-mass and 3-mass triangle integrals, the analytic results are actually not too complicated and would be well suited for inputs into any program for computing physically interesting quantities. One of the main goals of this paper is to obtain explicit analytic results for the rational parts of these two non-MHV helicity configurations. They are the last missing 
pieces for the complete partial helicity amplitudes of the 6-gluon one-loop QCD amplitude. Although our computation was done by hand, we have also input our analytic results into Mathematica codes. These codes would be useful for others to check our results and as inputs to compute physically interesting quantities.

This paper is organized as follows: in the next section we recall briefly the Feynman diagrams and the Feynman rules, tailored for the purpose of computing the rational parts of the 6-gluon helicity amplitude. A brief review of tensor reduction was given in Sect. 3. The next 4 sections of the paper present the results with some intermediate steps. In Sect. 8 we briefly discuss the factorization properties of the one-loop amplitudes and the checks done for the NMHV results. We collect some explicit results for the rational parts of Feynman integrals in the Appendix.

\section{Notation, the Feynman diagrams and the Feynman rules}

A word about notation: we use the same notation as given in [1]. We use

$\epsilon_{i(i+1) \cdots(i+n)}$ to denote the composite polarization vectors for sewing trees to the loop.

For the purpose of this paper we consider only the Feynman diagrams and Feynman rules for the one-loop gluon amplitude with scalars circulating in the loop. We do not follow the usual convention of differentiating different particles by different kinds of lines because there are only two kinds of particles: gluons and scalars, and scalars only appear in the loop.

For explicit calculation of the one-loop amplitude by the usual Feynman diagram technique, we can first collect all terms with the same loop structure into one entity. Generally a few cyclicly consecutive external lines are joined in tree diagrams and connected to the same point on the loop (sewing trees to loop). We denote the sum of all these contributions by $P_{i(i+1) \cdots(i+m-1)}$ for $m$ such external lines. For $m=1,2,3$, the relevant Feynman diagrams are shown in Fig. 1. Explicitly we have:

$$
\begin{aligned}
P_{i}(p) & =\left(\epsilon_{i}, p\right)=\left(\epsilon_{i}, p-k_{i}\right) \\
P_{i(i+1)}(p) & =\left(\epsilon_{i(i+1)}, p\right)-\frac{1}{2}\left(\epsilon_{i}, \epsilon_{i+1}\right)
\end{aligned}
$$




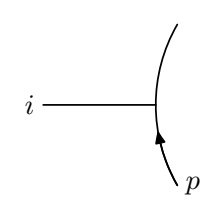

(a)
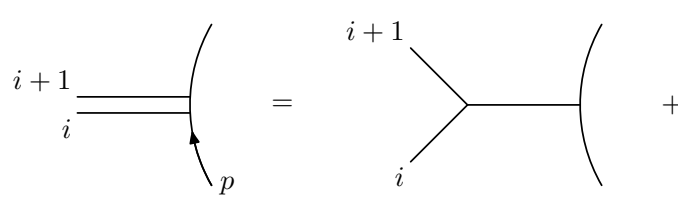

(b)

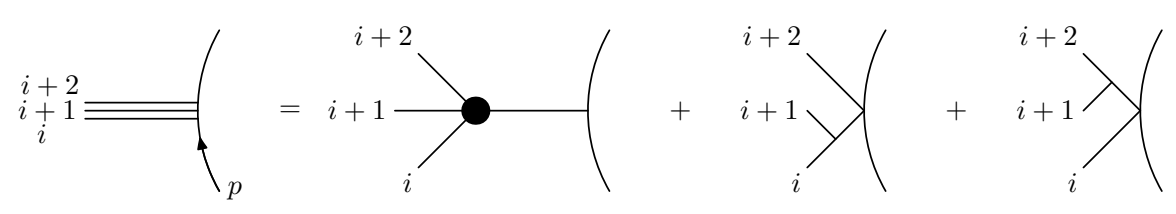

(c)

Figure 1: The Feynman rules for sewing trees to loop. The blob denotes an expansion of tree amplitude.

$$
P_{i(i+1)(i+2)}(p)=\left(\epsilon_{i(i+1)(i+2)}, p\right)-\frac{1}{2}\left(\left(\epsilon_{i(i+1)}, \epsilon_{i+2}\right)+\left(\epsilon_{i}, \epsilon_{(i+1)(i+2)}\right)\right),
$$

where $\epsilon \ldots$ 's are the composite polarization vectors introduced in [1]. The computation of these composite polarization vectors is a simplified version of the general recursive calculation of the tree-level $n$-gluon amplitudes [39].

Considering all these different tree diagrams just as the same diagram and denoting them by multiple parallel lines attached to the loop, we have only 51 different Feynman diagrams for the 6-gluon one-loop amplitude (with scalars circulating in the loop). Some representative diagrams are given in Fig. 2. The counting goes as follows:

- 1 hexagon diagram, the diagram (a);

- 6 pentagon diagrams because there are 6 different ways of combining two consecutive external lines, diagram (b) with $i=1, \cdots, 6$;

- 15 box diagrams which are further divided into 6 two-mass-hard box diagrams (diagram (c)), 3 two-mass-easy diagrams (diagram (d)) and 6 one-mass diagrams (diagram (e));

- 20 triangle diagrams: 2 three-mass triangle diagrams (diagrams (f) and (g)), $6+6$ two-mass triangle diagrams (diagrams (h) and (i)) and 6 one-mass triangle diagrams (diagram $(\mathrm{j}))$; 


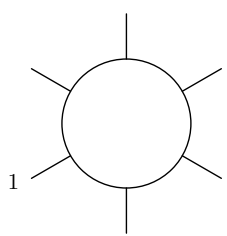

(a)

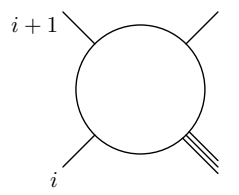

(e)

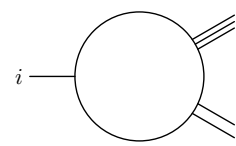

(i)

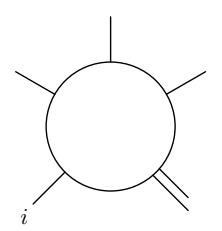

(b)

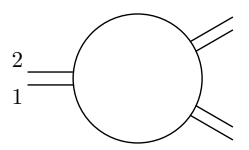

(f)

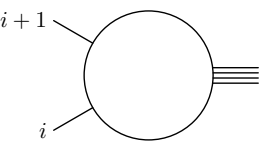

(j)

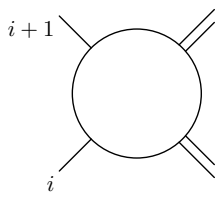

(c)

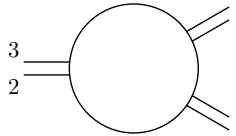

(g)

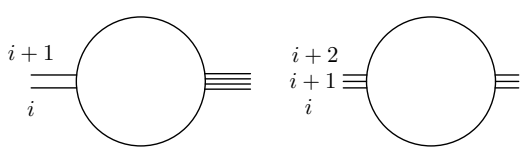

$(\mathrm{k})$

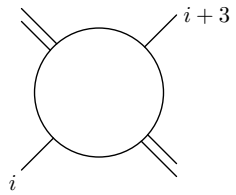

(d)

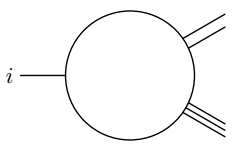

(l)

Figure 2: All the possible one-loop Feynman diagrams for six gluons. The index $i$ can run from 1 to 3 in (d) and (l), and 1 to 6 in the rests if there is an index $i$.

- 9 bubble diagrams (diagrams (k) and (l)).

We use the notation $I_{4}^{2 m h(i)}, I_{3}^{2 m(i)}$ and $\tilde{I}_{3}^{2 m(i)}$ to denote the rational parts of the Feynman integrals from the diagrams (c), (h) and (i). The rational parts from the diagrams (f) and (g) are denoted by $\tilde{I}_{3}^{3 m}$ and $I_{3}^{3 m}$ respectively. Explicit results from [1] are collected in the Appendix for quick reference.

It is straightforward to compute the rational part from each diagram. A better way to organize the computation is to use the simple tensor reduction formulas derive in [1]. To simplify things further we also organize the result into a summation over the symmetry group. This is possible if the choice of the reference momenta preserves the symmetry of the (rational part of the) amplitude. The results for different Feynman diagrams connected by symmetry operations can be obtained easily by using symmetry operations on the rational parts directly. In this way we need only to compute the different Feynman diagrams not connected by symmetry operations. This roughly reduces the number of different diagrams (needed to be computed 
explicitly) by a factor of the rank of the (finite) symmetry group. Even for Feynman diagrams which are invariant under the symmetry operations, it is also possible to split the results into serval pieces which are related by symmetry operations.

After we briefly review the tensor reduction formulas used in this paper in the next section, we will then present some intermediate steps and the results for the rational parts of the various (sets of) Feynman diagrams for the 4 different helicity configurations in 4 sections. At the beginning of each section we spell out the symmetry group of the helicity configurations. The final result for the rational part is written mostly as a summation over the symmetry group. We give only the explicit result for the identity group element. Others are obtained by symmetry actions. We will indicate which diagram(s) gives the relevant contributions. We also give the explicit choice of the polarization vectors for each helicity configuration. Our choice of the polarization vectors preserves the symmetry of the helicity configurations.

\section{Review of Tensor reduction of the one-loop amplitude}

There is a vast literature on this subject $[40,41,42,43,44,45,46]$. The tensor reduction relations we will use for our calculation of the 5- and 6-gluon amplitudes are quite simple. It is based on the BDK trick [34] of multiplying and dividing by spinor square roots. We purposely made the specific choice of the reference momenta in this paper to make all tensor reductions simple enough to obtain relatively compact analytic results for (the rational parts of) the 6-gluon amplitudes.

For tensor reductions with only 2 neighboring same helicity external gluons, it is possible to choose the reference momenta to be each other's mo-

menta, i.e. $\epsilon_{1}=\lambda_{1} \tilde{\lambda}_{2}, \epsilon_{2}=\lambda_{2} \tilde{\lambda}_{1}$. The tensor reduction is done by considering the contributions from 2 diagrams together and the result formula is shown pictorially in Fig. 3. The exact algebraic formula is:

$$
\begin{aligned}
\frac{\left(\epsilon_{1}, p+k_{1}\right)\left(\epsilon_{2}, p\right)}{\left(p+k_{1}\right)^{2} p^{2}\left(p-k_{2}\right)^{2}} & +\frac{\left(\epsilon_{12}, p+k_{1}\right)-\left(\epsilon_{1}, \epsilon_{2}\right) / 2}{\left(p+k_{1}\right)^{2}\left(p-k_{2}\right)^{2}} \\
& =-\frac{1}{p^{2}}+\frac{1 / 2}{\left(p+k_{1}\right)^{2}}+\frac{1 / 2}{\left(p-k_{2}\right)^{2}}
\end{aligned}
$$




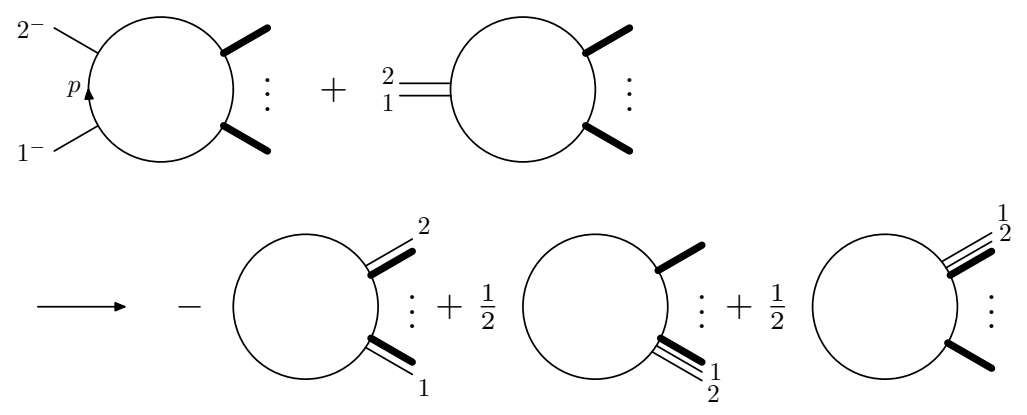

Figure 3: For two adjacent same helicities, the tensor reduction for the combination of two diagrams is even simpler.

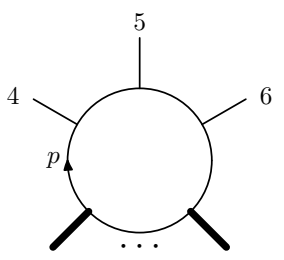

(a)

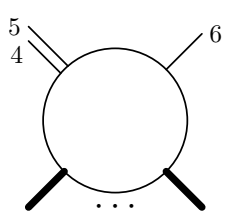

(b)

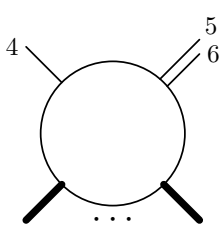

(c)

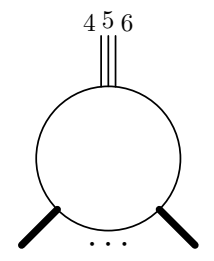

(d)

Figure 4: For three adjacent same helicities, the tensor reduction for the combination of these four diagrams is also quite simple.

For three adjacent particles with the same helicity, we choose the following polarization vectors (omitting an overall factor for each polarization vector):

$$
\epsilon_{4}=\lambda_{5} \tilde{\lambda}_{4}, \quad \epsilon_{5}=\eta \tilde{\lambda}_{5}, \quad \epsilon_{6}=\lambda_{5} \tilde{\lambda}_{6} .
$$

Then the tensor reduction of the 4 diagrams shown in Fig. 4 is given as follows:

$$
\begin{aligned}
A_{a}+A_{b}+A_{c}+A_{d} & =\frac{\langle\eta 5\rangle}{2\langle 65\rangle} \frac{P_{4}(p)}{p^{2}\left(p-k_{4}\right)^{2}} \\
& +\frac{\langle\eta 5\rangle}{2\langle 45\rangle} \frac{P_{6}\left(p-k_{45}\right)}{\left(p-k_{45}\right)^{2}\left(p-k_{456}\right)^{2}} .
\end{aligned}
$$

which has a nice symmetric property under the flipping operation $4 \leftrightarrow 6$.

Sometimes we also need the reduction formulas for $A_{a}+A_{b}$ or $A_{a}+A_{c}$ :

$$
A_{a}+A_{b}=\frac{P_{4}(p) P_{5}\left(p-k_{4}\right)}{p^{2}\left(p-k_{4}\right)^{2}\left(p-k_{45}\right)^{2}}+\frac{P_{45}(p)}{p^{2}\left(p-k_{45}\right)^{2}}
$$




$$
\begin{aligned}
& =-\frac{\left(\eta \tilde{\lambda}_{4}, p\right)}{p^{2}\left(p-k_{4}\right)^{2}}+\frac{\langle\eta 5\rangle}{2\langle 45\rangle}\left[\frac{1}{\left(p-k_{45}\right)^{2}}-\frac{1}{p^{2}}\right], \\
A_{a}+A_{c} & =\frac{P_{5}(\tilde{p}) P_{6}\left(\tilde{p}-k_{5}\right)}{\tilde{p}^{2}\left(\tilde{p}-k_{5}\right)^{2}\left(\tilde{p}-k_{56}\right)^{2}}+\frac{P_{56}(\tilde{p})}{\tilde{p}^{2}\left(\tilde{p}-k_{56}\right)^{2}} \\
& =\frac{\left(\eta \tilde{\lambda}_{6}, \tilde{p}-k_{5}\right)}{\left(\tilde{p}-k_{5}\right)^{2}\left(\tilde{p}-k_{56}\right)^{2}}+\frac{\langle\eta 5\rangle}{2\langle 65\rangle}\left[\frac{1}{\tilde{p}^{2}}-\frac{1}{\left(\tilde{p}-k_{56}\right)^{2}}\right],
\end{aligned}
$$

where $\tilde{p}=p-k_{4}$.

\section{$4 \quad$ MHV: $R\left(1^{-} 2^{+} 3^{+} 4^{-} 5^{+} 6^{+}\right)$}

The reader is referred to eq. (31) in [1] for the precise definition of the rational part, and it is the same to the next three sections. For this helicity configuration, the symmetry group is

$$
G=\{1, \sigma, \tau, \sigma \tau\}
$$

where $\sigma=(2 \leftrightarrow 6,3 \leftrightarrow 5)$ and $\tau=(i \rightarrow i+3)$ are the two generators of the symmetry group. We have $\sigma \tau=(1 \leftrightarrow 4,2 \leftrightarrow 3,5 \leftrightarrow 6)$. Under the symmetry action $\sigma$ (or $\sigma \tau$ ) the ordering of the external lines is reversed. The reversed ordering can be easily transformed to the standard ordering (clockwise in our convention) by using the symmetric or anti-symmetric property of the composite polarization vectors. From this change of the ordering, there is an overall sign of $(-)^{n}$ for an $n$-point amplitude which matches the same factor from the reversing of the ordering of the color factor.

The polarization vectors for this helicity configuration can be chosen as follows:

$$
\begin{aligned}
\epsilon_{2} & =\frac{\lambda_{3} \tilde{\lambda}_{2}}{\langle 32\rangle}, & \epsilon_{3} & =\frac{\lambda_{2} \tilde{\lambda}_{3}}{\langle 23\rangle}, \\
\epsilon_{5} & =\frac{\lambda_{6} \tilde{\lambda}_{5}}{\langle 65\rangle}, & \epsilon_{6} & =\frac{\lambda_{5} \tilde{\lambda}_{6}}{\langle 56\rangle} .
\end{aligned}
$$

We can leave the reference momenta of $\epsilon_{1,4}$ arbitrary. To be specific, an explicit symmetric choice is $\epsilon_{1}=\frac{\lambda_{1} \tilde{\lambda}_{4}}{[14]}$ and $\epsilon_{4}=\frac{\lambda_{4} \tilde{\lambda}_{1}}{[41]}$. To ease the writing, we will omit all the denominators of these polarization vectors and simply use $\epsilon_{2}=\lambda_{3} \tilde{\lambda}_{2}$, etc. The overall factor will be reinstated in the final result. 
By using the above symmetry group $G$, the 51 Feynman diagrams are classified into 10 sets. Representatives (or all Feynman diagrams) from each set are shown in Figs. 5 to 14. The diagrams are ordered from higher point diagrams to lower point diagrams because the tensor reduction of higher point diagrams often gives pieces which are cancelled by lower point diagrams. Our strategy is to make this (local) cancellation manifest by grouping these diagrams together. We now compute the rational part of each set of diagrams in turn.

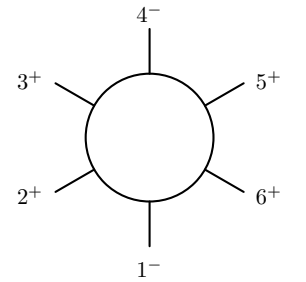

(a)

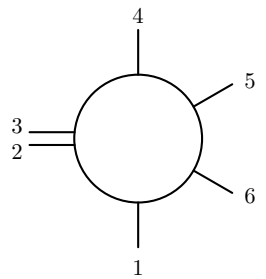

(b)

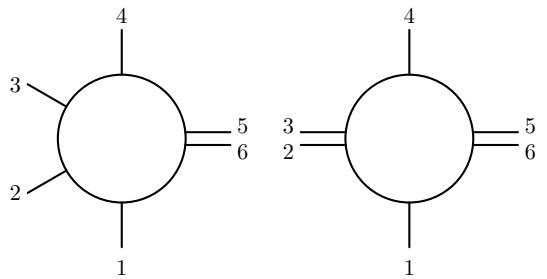

(c)

(d)

Figure 5: These 4 diagrams are tensor reduced simply with the two pairs of external lines $k_{2,3}$ and $k_{5,6}$ with the same helicities.

The 1st set consists of 4 Feynman diagrams as shown in Fig. 5. The rational part can be computed quite easily by using the tensor reduction with the two pairs of external lines $k_{2,3}$ and $k_{5,6}$ with the same helicities. The Feynman diagrams reduce to only bubble diagrams. The rational part is:

$$
\begin{aligned}
R_{1} & =-\frac{1}{72}\left(4\left(\epsilon_{1}, k_{612}\right)\left(\epsilon_{4}, k_{345}\right)+2 \sum_{G}\left(\epsilon_{1}, k_{12}\right)\left(\epsilon_{4}, k_{12}\right)\right. \\
& \left.+\left(\epsilon_{1}, k_{123}\right)\left(\epsilon_{4}, k_{456}\right)+\left(\epsilon_{1}, k_{561}\right)\left(\epsilon_{4}, k_{234}\right)\right) \\
& +\frac{1}{36}\left(2\left(s_{12}+s_{34}+s_{45}+s_{61}\right)-4 s_{345}-s_{123}-s_{234}\right)\left(\epsilon_{1}, \epsilon_{4}\right) .
\end{aligned}
$$

The correction terms are cancelled between the 3 diagrams obtained from the 1st step of the tensor reduction from hexagon (+ pentagon) to box. By analyzing the symmetry property of the various terms, we can write this result as a summation over the symmetry group G. Explicitly we have

$$
R_{1}(1)=-\frac{1}{36}\left(\left(\epsilon_{1}, k_{2}\right)\left(\epsilon_{4}, k_{12}\right)+\frac{1}{2}\left(\epsilon_{1}, k_{612}\right)\left(\epsilon_{4}, k_{345}\right)\right.
$$




$$
\begin{aligned}
& \left.+\frac{1}{4}\left(\epsilon_{1}, k_{123}\right)\left(\epsilon_{4}, k_{456}\right)\right)+\frac{1}{72}\left(4 s_{12}-2 s_{345}-s_{123}\right)\left(\epsilon_{1}, \epsilon_{4}\right) \\
R_{1}(g) & =g\left(R_{1}(1)\right) \\
R_{1} & =\sum_{g \in G} R_{1}(g)
\end{aligned}
$$

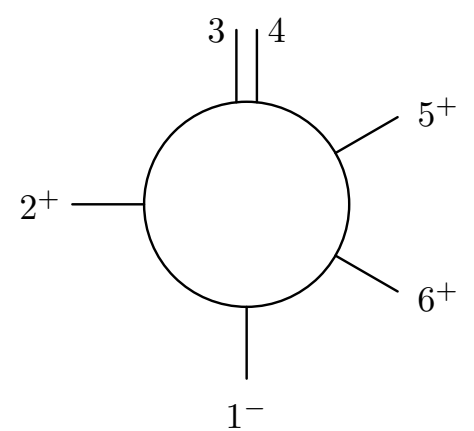

(a)

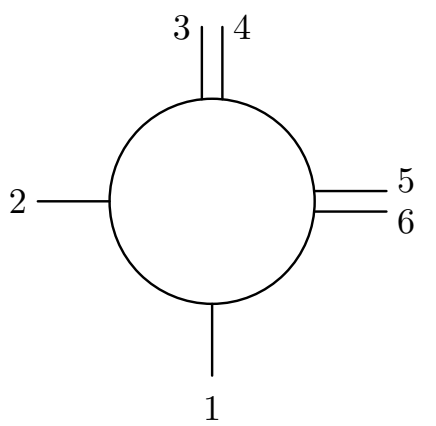

(b)

Figure 6: This set has 8 diagrams. The other 6 diagrams are obtained by symmetry operations. The above 2 diagrams can be reduced simply with $k_{5,6}$.

The 2 nd set consists of 8 diagrams and two representatives are shown in Fig. 6. The rational part from these two diagrams gives $R_{2}(1)$ and we have:

$$
\begin{aligned}
R_{2}(1) & =\frac{\left(\epsilon_{3}, \epsilon_{4}\right)\left(\epsilon_{1}, k_{2}\right)}{8}\left(\frac{2\left(\epsilon_{2}, k_{61}\right)}{s_{61}-s_{345}}-\frac{\left(\epsilon_{2}, k_{34}\right)}{s_{34}-s_{234}}+\frac{\left(\epsilon_{2}, k_{1}\right)}{s_{12}}\right) \\
& -\frac{1}{18}\left(\left(\epsilon_{2}, \epsilon_{34}\right) \epsilon_{1}+\left(\epsilon_{2}, \epsilon_{1}\right) \epsilon_{34}+\left(\epsilon_{1}, \epsilon_{34}\right) \epsilon_{2}, k_{5}-k_{6}\right) \\
& -\frac{1}{24}\left(\left(\epsilon_{2}, \epsilon_{34}\right)\left(\epsilon_{1}, k_{2}\right)+\left(\epsilon_{2}, \epsilon_{1}\right)\left(\epsilon_{34}, k_{2}\right)\right) \\
& +\frac{1}{12}\left(\epsilon_{2}, \epsilon_{1}\right)\left(\epsilon_{34}, k_{1}+2 k_{2}\right) \\
& -\frac{s_{61}+s_{345}}{6\left(s_{61}-s_{345}\right)^{2}}\left(\epsilon_{2}, k_{61}\right)\left(\epsilon_{34}, k_{2}\right)\left(\epsilon_{1}, k_{2}\right) \\
& -\frac{s_{34}+s_{234}}{12\left(s_{34}-s_{234}\right)^{2}}\left(\epsilon_{2}, k_{34}\right)\left(\epsilon_{34}, k_{2}\right)\left(\epsilon_{1}, k_{2}\right) \\
& +\frac{\left(\epsilon_{2}, k_{61}\right)}{6\left(s_{61}-s_{345}\right)}\left(\left(\epsilon_{34}, k_{2}\right)\left(\epsilon_{1}, k_{6}\right)-\left(\epsilon_{34}, k_{5}\right)\left(\epsilon_{1}, k_{2}\right)\right)
\end{aligned}
$$




$$
\begin{aligned}
& -\frac{\left(\epsilon_{2}, k_{34}\right)}{12\left(s_{34}-s_{234}\right)}\left(\epsilon_{34}, k_{2}\right)\left(\epsilon_{1}, k_{56}\right) \\
& -\frac{s_{61}+s_{345}}{12\left(s_{61}-s_{345}\right)}\left(\left(\epsilon_{2}, \epsilon_{34}\right)\left(\epsilon_{1}, k_{2}\right)+\left(\epsilon_{2}, \epsilon_{1}\right)\left(\epsilon_{34}, k_{2}\right)\right) \\
& -\frac{s_{34}+s_{234}}{24\left(s_{34}-s_{234}\right)}\left(\left(\epsilon_{2}, \epsilon_{34}\right)\left(\epsilon_{1}, k_{2}\right)+\left(\epsilon_{2}, \epsilon_{1}\right)\left(\epsilon_{34}, k_{2}\right)\right) .
\end{aligned}
$$

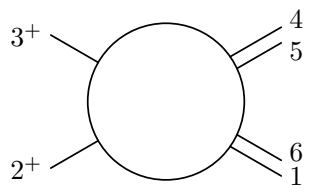

(a)

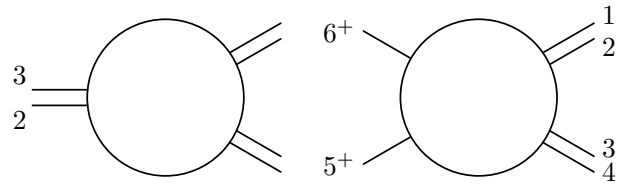

(b)

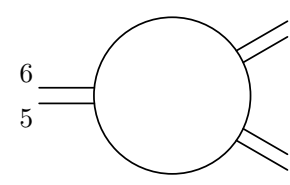

(d)

Figure 7: This set has 4 diagrams. The first 2 diagrams and the last 2 are related by the symmetry operation $\tau$.

The computation of the rational part from the 4 diagrams shown in Fig. 7 is also easy by using the tensor reduction with $k_{2,3}$ or $k_{5,6}$. Here we need a correction term to the naive tensor reduction in $D=4$ (see [1] for explicit computation of the correction term). The result is:

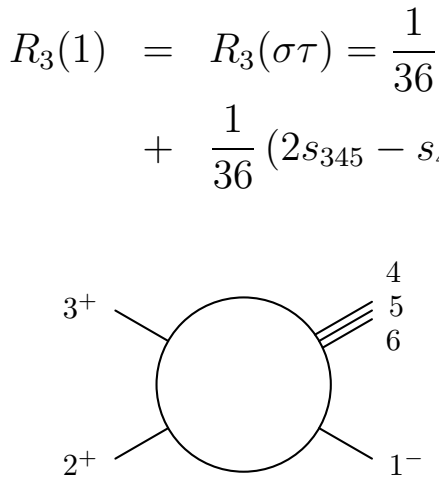

(a)

Figure 8: This set has 8 diagrams. The other 6 diagrams are obtained by symmetry operations.

The tensor reduction for the two diagrams shown in Fig. 8 is also easy with the 2 external momenta $k_{2,3}$ because of the same adjacent helicities. 
They are reduced to bubble diagrams. As in the last case with the reduction of the 2-mass-hard diagram, we also need a correction term to the naive tensor reduction in $D=4$. By including the correction term we have

$$
R_{4}(1)=\frac{1}{18}\left(\epsilon_{1}, k_{2}\right)\left(\epsilon_{456}, k_{3}\right)+\frac{1}{18}\left(2 s_{12}-s_{123}-3 s_{23}\right)\left(\epsilon_{1}, \epsilon_{456}\right) .
$$

In the above we have used the physical condition of the (composite) polarization vectors. The term with the factor $s_{23}$ comes from the correction term in the tensor reduction from the (one-mass) box diagram. The other 6 diagrams (in 3 pairs) give the contributions $R_{4}(\sigma), R_{4}(\tau)$ and $R_{4}(\sigma \tau)$.

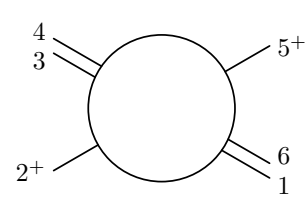

(a)

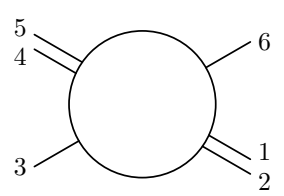

(b)

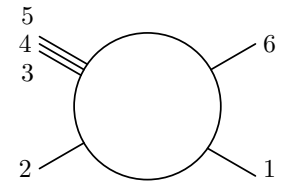

(c)

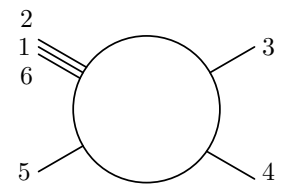

(d)

Figure 9: This set has 4 diagrams. (a) and (b) are related by symmetry operation $\sigma$, whereas (c) and (d) are related by symmetry operation $\tau$.

The 4 diagrams shown in Fig. 9 give the most complicated algebraic expressions for this helicity configuration. We give the results in 2 parts:

$$
\begin{aligned}
R_{5,1}(1) & =\frac{\langle 26\rangle}{4\langle 25\rangle}\left[\frac{\left(\epsilon_{2}, k_{34}\right)}{s_{34}-s_{234}}-\frac{\left(\epsilon_{2}, k_{61}\right)}{s_{61}-s_{345}}\right]\left(\epsilon_{3}, \epsilon_{4}\right)\left(\epsilon_{61}, k_{2}\right) \\
& +\frac{\langle 26\rangle}{4\langle 25\rangle}\left[\frac{\left(\epsilon_{2}, k_{34}\right)}{s_{34}-s_{234}}-\frac{\left(\epsilon_{2}, k_{61}\right)}{s_{61}-s_{345}}\right]\left(\epsilon_{1}, \epsilon_{6}\right)\left(\epsilon_{34}, k_{2}\right) \\
& +\frac{\langle 25\rangle}{4\langle 26\rangle}\left[\frac{\left(\epsilon_{2}, k_{61}\right)}{s_{61}-s_{345}}+\frac{\left(\epsilon_{2}, k_{1}\right)}{s_{12}}\right]\left(\epsilon_{1}, k_{2}\right)\left(\left(\epsilon_{3}, \epsilon_{45}\right)+\left(\epsilon_{34}, \epsilon_{5}\right)\right) \\
& +\frac{\langle 26\rangle}{9\langle 25\rangle}\left(\left(\epsilon_{2}, \epsilon_{34}\right) \epsilon_{61}+\left(\epsilon_{2}, \epsilon_{61}\right) \epsilon_{34}+\left(\epsilon_{34}, \epsilon_{61}\right) \epsilon_{2}, k_{5}\right) \\
& +\frac{\langle 25\rangle}{\langle 26\rangle}\left[\frac{1}{9}\left(\left(\epsilon_{2}, \epsilon_{345}\right) \epsilon_{1}+\left(\epsilon_{2}, \epsilon_{1}\right) \epsilon_{345}+\left(\epsilon_{345}, \epsilon_{1}\right) \epsilon_{2}, k_{6}\right)\right. \\
& \left.-\frac{1}{12}\left(\left(\epsilon_{2}, \epsilon_{345}\right) \epsilon_{1}+\left(\epsilon_{2}, \epsilon_{1}\right) \epsilon_{345}, k_{2}\right)+\frac{1}{6}\left(\epsilon_{1}, \epsilon_{2}\right)\left(\epsilon_{345}, k_{2}-k_{6}\right)\right] \\
& +\frac{\langle 26\rangle}{\langle 25\rangle}\left[\frac{1}{12}\left[\frac{s_{34}+s_{234}}{s_{34}-s_{234}}+\frac{s_{61}+s_{345}}{s_{61}-s_{345}}\right]\left(\left(\epsilon_{2}, \epsilon_{34}\right) \epsilon_{61}+\left(\epsilon_{2}, \epsilon_{61}\right) \epsilon_{34}, k_{2}\right)\right.
\end{aligned}
$$




$$
\begin{aligned}
& +\left(\epsilon_{34}, k_{2}\right)\left(\epsilon_{61}, k_{2}\right)\left[\frac{\left(s_{34}+s_{234}\right)\left(\epsilon_{2}, k_{34}\right)}{6\left(s_{34}-s_{234}\right)^{2}}+\frac{\left(s_{61}+s_{345}\right)\left(\epsilon_{2}, k_{61}\right)}{6\left(s_{61}-s_{345}\right)^{2}}\right] \\
& \left.+\left[\frac{\left(\epsilon_{2}, k_{34}\right)\left(\epsilon_{34}, k_{2}\right)\left(\epsilon_{61}, k_{5}\right)}{6\left(s_{34}-s_{234}\right)}+\frac{\left(\epsilon_{2}, k_{61}\right)\left(\epsilon_{34}, k_{5}\right)\left(\epsilon_{61}, k_{2}\right)}{6\left(s_{61}-s_{345}\right)}\right]\right] \\
& -\frac{\langle 25\rangle}{\langle 26\rangle}\left[\frac{s_{61}+s_{345}}{12\left(s_{61}-s_{345}\right)}\left(\left(\epsilon_{2}, \epsilon_{345}\right) \epsilon_{1}+\left(\epsilon_{2}, \epsilon_{1}\right) \epsilon_{345}, k_{2}\right)\right. \\
& \left.+\left(\epsilon_{2}, k_{61}\right)\left(\epsilon_{345}, k_{2}\right)\left[\frac{\left(s_{61}+s_{345}\right)\left(\epsilon_{1}, k_{2}\right)}{6\left(s_{61}-s_{345}\right)^{2}}-\frac{\left(\epsilon_{1}, k_{6}\right)}{6\left(s_{61}-s_{345}\right)}\right]\right],
\end{aligned}
$$

and

$$
\begin{aligned}
R_{5,2}(1)= & \left(k_{2}, k_{5}\right)\left(\epsilon_{34}, \epsilon_{61}\right)\left[\frac{5}{18} \frac{\langle 23\rangle\langle 56\rangle}{\langle 25\rangle^{2}}-\frac{1}{18} \frac{\langle 35\rangle\langle 26\rangle}{\langle 25\rangle^{2}}\right] \\
+ & \left(\left(\epsilon_{34}, k_{2}\right)\left(\epsilon_{61}, k_{5}\right)+\left(\epsilon_{34}, k_{5}\right)\left(\epsilon_{61}, k_{2}\right)\right) \\
& \times\left[-\frac{2}{9} \frac{\langle 23\rangle\langle 56\rangle}{\langle 25\rangle^{2}}+\frac{1}{36} \frac{\langle 35\rangle\langle 26\rangle}{\langle 25\rangle^{2}}\right] \\
+ & \left(k_{2}, k_{6}\right)\left(\epsilon_{1}, \epsilon_{345}\right)\left[-\frac{5}{18} \frac{\langle 23\rangle\langle 56\rangle}{\langle 26\rangle^{2}}-\frac{1}{18} \frac{\langle 36\rangle\langle 25\rangle}{\langle 26\rangle^{2}}\right] \\
+ & \left(\epsilon_{1}, k_{2}\right)\left(\epsilon_{345}, k_{6}\right)\left[\frac{4}{9} \frac{\langle 23\rangle\langle 56\rangle}{\langle 26\rangle^{2}}+\frac{1}{18} \frac{\langle 36\rangle\langle 25\rangle}{\langle 26\rangle^{2}}\right] \\
+ & \left(\epsilon_{1}, k_{2}\right)\left(\epsilon_{345}, k_{2}\right) \frac{\langle 23\rangle\langle 56\rangle}{4\langle 26\rangle^{2}}+\frac{s_{61}+s_{345}}{s_{61}-s_{345}} \\
& \times\left[\frac{\langle 23\rangle\langle 56\rangle}{4\langle 25\rangle^{2}}\left(\epsilon_{34}, k_{2}\right)\left(\epsilon_{61}, k_{2}\right)+\frac{\langle 23\rangle\langle 56\rangle}{4\langle 26\rangle^{2}}\left(\epsilon_{345}, k_{2}\right)\left(\epsilon_{1}, k_{2}\right)\right] \\
+ & \frac{s_{34}+s_{234}}{s_{34}-s_{234}} \frac{\langle 23\rangle\langle 56\rangle}{4\langle 25\rangle^{2}}\left(\epsilon_{34}, k_{2}\right)\left(\epsilon_{61}, k_{2}\right) .
\end{aligned}
$$

The complete rational part is $R_{5}(1)=R_{5,1}(1)+R_{5,2}(1)$.

The rational part from the 2-mass triangle diagrams shown in Fig. 10 can be written directly by using eqs. (106) and (107). We have

$$
\begin{aligned}
R_{6,1}(1) & =\frac{1}{36}\left(7\left(\epsilon_{2}, \epsilon_{34}\right)\left(\epsilon_{561}, k_{2}\right)-7\left(\epsilon_{2}, \epsilon_{561}\right)\left(\epsilon_{34}, k_{2}\right)+4\left(\epsilon_{34}, \epsilon_{561}\right)\left(\epsilon_{2}, k_{34}\right)\right) \\
& -\frac{s_{34}+s_{234}}{12\left(s_{34}-s_{234}\right)}\left(\left(\epsilon_{2}, \epsilon_{34}\right)\left(\epsilon_{561}, k_{2}\right)+\left(\epsilon_{2}, \epsilon_{561}\right)\left(\epsilon_{34}, k_{2}\right)\right)
\end{aligned}
$$




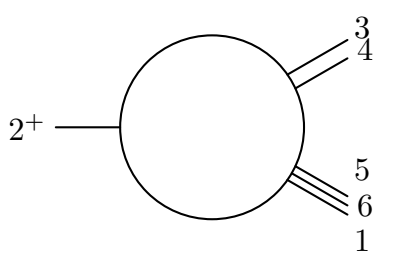

(a)

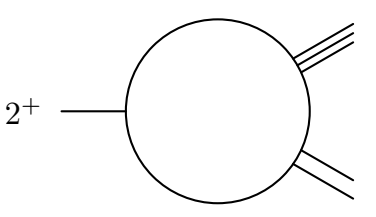

(b)

Figure 10: This set has 8 diagrams. Others are obtained from the above 2 diagrams by symmetry operations.

$$
\begin{aligned}
- & \frac{s_{34}+s_{234}}{6\left(s_{34}-s_{234}\right)^{2}}\left(\epsilon_{2}, k_{34}\right)\left(\epsilon_{34}, k_{2}\right)\left(\epsilon_{561}, k_{2}\right) \\
& -\frac{1}{4}\left(\epsilon_{3}, \epsilon_{4}\right)\left[\left(\epsilon_{2}, \epsilon_{561}\right)+\frac{\left(\epsilon_{2}, k_{34}\right)\left(\epsilon_{561}, k_{2}\right)}{s_{34}-s_{234}}\right] \\
& -\frac{1}{4}\left(\left(\epsilon_{5}, \epsilon_{61}\right)+\left(\epsilon_{56}, \epsilon_{1}\right)\right)\left[\left(\epsilon_{2}, \epsilon_{34}\right)+\frac{\left(\epsilon_{2}, k_{34}\right)\left(\epsilon_{34}, k_{2}\right)}{s_{34}-s_{234}}\right], \\
R_{6,2}(1)= & \frac{1}{36}\left(7\left(\epsilon_{2}, \epsilon_{345}\right)\left(\epsilon_{61}, k_{2}\right)-7\left(\epsilon_{2}, \epsilon_{61}\right)\left(\epsilon_{345}, k_{2}\right)-4\left(\epsilon_{345}, \epsilon_{61}\right)\left(\epsilon_{2}, k_{61}\right)\right) \\
+ & \frac{s_{61}+s_{345}}{12\left(s_{61}-s_{345}\right)}\left(\left(\epsilon_{2}, \epsilon_{345}\right)\left(\epsilon_{61}, k_{2}\right)+\left(\epsilon_{2}, \epsilon_{61}\right)\left(\epsilon_{345}, k_{2}\right)\right) \\
+ & \frac{s_{61}+s_{345}}{6\left(s_{61}-s_{345}\right)^{2}}\left(\epsilon_{2}, k_{61}\right)\left(\epsilon_{345}, k_{2}\right)\left(\epsilon_{61}, k_{2}\right) \\
- & \frac{1}{4}\left(\epsilon_{6}, \epsilon_{1}\right)\left[\left(\epsilon_{2}, \epsilon_{345}\right)+\frac{\left(\epsilon_{2}, k_{61}\right)\left(\epsilon_{345}, k_{2}\right)}{s_{61}-s_{345}}\right] \\
& -\frac{1}{4}\left(\left(\epsilon_{3}, \epsilon_{45}\right)+\left(\epsilon_{34}, \epsilon_{5}\right)\right)\left[\left(\epsilon_{2}, \epsilon_{61}\right)+\frac{\left(\epsilon_{2}, k_{61}\right)\left(\epsilon_{61}, k_{2}\right)}{s_{61}-s_{345}}\right], \\
R_{6}(1)= & R_{6,1}(1)+R_{6,2}(1) .
\end{aligned}
$$

The diagrams in Fig. 11 can be easily computed either directly or by using the tensor reduction formula with correction terms. We have:

$$
\begin{aligned}
R_{7}(1)=R_{7}(\sigma \tau) & =\frac{1}{8} s_{23}\left(\left(\epsilon_{45}, \epsilon_{61}\right)+\left(\epsilon_{4}, \epsilon_{561}\right)+\left(\epsilon_{456}, \epsilon_{1}\right)\right) \\
& -\frac{1}{12} s_{23}\left(\epsilon_{4561}, k_{2}\right) .
\end{aligned}
$$




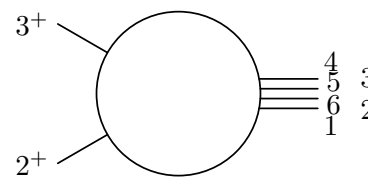

(a)

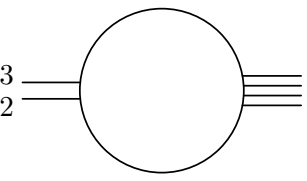

(b)

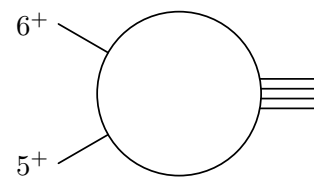

(c)

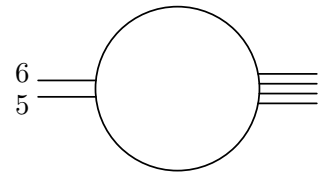

(d)

Figure 11: This set also has 4 diagrams. The last 2 are obtained from the first two diagrams by the $\tau$ symmetry operation.

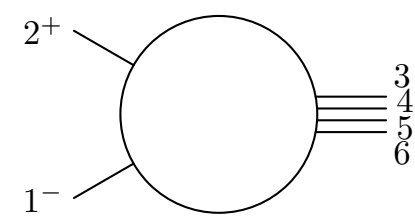

(a)

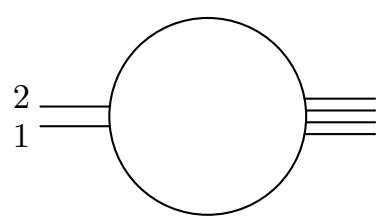

(b)

Figure 12: This set has 8 diagrams. The other 6 diagrams are obtained from these two diagrams by symmetry operations. The rational part from diagram (a) cancels the rational part from diagram (b).

The rational parts from the two diagrams in Fig. 12 have a very nice property. By using the relation $\left(\epsilon_{1}, k_{2}\right)\left(\epsilon_{2}, k_{1}\right)=s_{12}\left(\epsilon_{1}, \epsilon_{2}\right)$ for a pair of neighboring lines with opposite helicities, one shows that they cancel each other and the sum of the rational parts is identically equal to zero, i.e.,

$$
R_{8}(1)=0
$$

This result is also true for the other helicity configurations as long as the 2 massless external lines in the 1-mass triangle diagram have opposite helicities.

For the diagrams in Fig. 13, we have

$$
\begin{aligned}
& R_{9}(1)=R_{9}(\tau)=-\frac{1}{18} s_{123}\left(\epsilon_{123}, \epsilon_{456}\right) \\
& R_{9}(\sigma)=R_{9}(\sigma \tau)=\left.R_{9}(1)\right|_{2 \leftrightarrow 6,3 \leftrightarrow 5}=-\frac{1}{18} s_{234}\left(\epsilon_{234}, \epsilon_{561}\right) .
\end{aligned}
$$

In the above, the indicated permutations are applied to the polarization vectors, momenta and kinematic variables. 


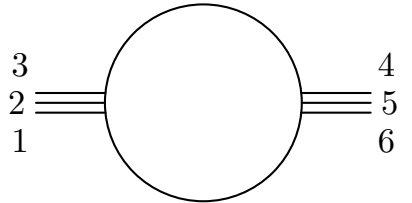

(a)

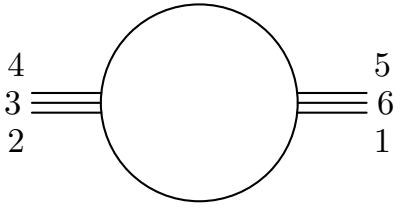

(b)

Figure 13: This set has 2 diagrams. The 2 diagrams are related by the $\sigma$ symmetry operation.

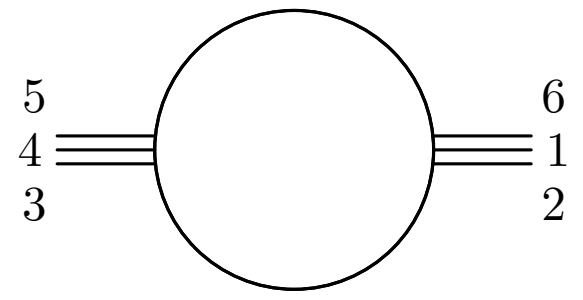

Figure 14: This diagram is the most symmetric one. It leads to a rational part which is invariant under all symmetric operations.

The diagram shown in Fig. 14 is invariant under all symmetry operations. The rational part is:

$$
R_{10}=-\frac{1}{9} s_{345}\left(\epsilon_{345}, \epsilon_{612}\right) .
$$

Dividing by the rank of the (finite) symmetry group gives $R_{10}(g)$ :

$$
R_{10}(g)=-\frac{1}{36} s_{345}\left(\epsilon_{345}, \epsilon_{612}\right), \quad g=1, \sigma, \tau, \text { or } \sigma \tau .
$$

Combining all the above results together, the complete rational part is given as follows:

$$
R=F \sum_{g \in G} R(g)=F \sum_{g \in G} \sum_{i=1}^{10} R_{i}(g),
$$

where

$$
F=-\frac{1}{(\langle 23\rangle\langle 56\rangle[14])^{2}},
$$

is an overall factor from the omitted factors of the polarization vectors. The explicit form of $R$ is not quite illuminating and we will not write it down here. 
To be sure that the above result is correct we have chosen another completely different set of reference momenta for the polarization vectors and obtained the same analytic result (in a quite different form of course). To do some consistency checks, let us consider the double pole terms from $R(1)$ :

$$
\begin{aligned}
A_{2}(1) & =\frac{s_{61}+s_{345}}{6\left(s_{61}-s_{345}\right)^{2}}\left[\left(\epsilon_{2}, k_{61}\right)\left(\epsilon_{345}, k_{2}\right)\left(\epsilon_{61}, k_{2}\right)-\left(\epsilon_{2}, k_{61}\right)\left(\epsilon_{34}, k_{2}\right)\left(\epsilon_{1}, k_{2}\right)\right. \\
& \left.-\frac{\langle 25\rangle}{\langle 26\rangle}\left(\epsilon_{2}, k_{61}\right)\left(\epsilon_{345}, k_{2}\right)\left(\epsilon_{1}, k_{2}\right)+\frac{\langle 26\rangle}{\langle 25\rangle}\left(\epsilon_{34}, k_{2}\right)\left(\epsilon_{61}, k_{2}\right)\left(\epsilon_{2}, k_{61}\right)\right] \\
& +\frac{\left(s_{34}+s_{234}\right)}{6\left(s_{34}-s_{234}\right)^{2}}\left[\frac{\langle 26\rangle}{\langle 25\rangle}\left(\epsilon_{34}, k_{2}\right)\left(\epsilon_{61}, k_{2}\right)\left(\epsilon_{2}, k_{34}\right)\right. \\
& \left.-\frac{1}{2}\left(\epsilon_{2}, k_{34}\right)\left(\epsilon_{34}, k_{2}\right)\left(\epsilon_{1}, k_{2}\right)-\left(\epsilon_{2}, k_{34}\right)\left(\epsilon_{34}, k_{2}\right)\left(\epsilon_{561}, k_{2}\right)\right] .
\end{aligned}
$$

We have checked that these terms agree with the result of the cut-constructible part [26] ( $T_{3}$ terms) as required by the absence of spurious poles in the complete amplitude (cut-constructible part + rational part), up to simple pole terms. These residual simple pole terms added together with the simple pole terms from our explicit computations also agree with the cut-constructible part of $[26]$ ( $T_{2}$ terms).

In comparing with the notation of Bern, Dixon and Kosower [6], $R$ is one half of what they called $R(z)$. The exact relation is:

$$
R=\frac{1}{2}\left(\left.R(z)\right|_{z=0}+\frac{2}{9} A^{\text {tree }}\right)
$$

by striping away a factor of $i c_{\Gamma}$. By using the Mathematica code provided by them for their result, we have done a numerical check by comparing our result with the result of Berger, Bern, Dixon, Forde and Kosower [9]. The check is done by randomly assigning a set of complex rational numbers to the 12 independent $\langle i j\rangle$ and $[i j]$. We found exact match without suffering the usual real number approximation because all computations are done with rational numbers.

We note that this numerical check actually gives a proof of the equivalence of our result and the result of [9]. This is because a rational function with a limited number of different denominators and a fixed degree can only depends on a finite number of free parameters. Although we have no exact estimation of the number of these free parameters, the number (over a few thousands) of checks we have done should be quite enough. 


\section{MHV: $R\left(1^{-} 2^{+} 3^{-} 4^{+} 5^{+} 6^{+}\right)$}

For this helicity configuration, the symmetry group is

$$
G=\{1, \sigma\}
$$

where $\sigma=(1 \leftrightarrow 3,4 \leftrightarrow 6)$. The polarization vectors are:

$$
\begin{array}{ll}
\epsilon_{1}=\frac{\lambda_{1} \tilde{\lambda}_{2}}{[12]}, & \epsilon_{3}=\frac{\lambda_{3} \tilde{\lambda}_{2}}{[32]}, \\
\epsilon_{2}=\frac{\lambda_{5} \tilde{\lambda}_{2}}{\langle 52\rangle}, & \epsilon_{5}=\frac{\lambda_{2} \tilde{\lambda}_{5}}{\langle 25\rangle}, \\
\epsilon_{4}=\frac{\lambda_{5} \tilde{\lambda}_{4}}{\langle 54\rangle}, & \epsilon_{6}=\frac{\lambda_{5} \tilde{\lambda}_{6}}{\langle 56\rangle} .
\end{array}
$$

We note that the above choice of reference momenta preserves the symmetry of the amplitude manifestly.

For this helicity configuration, there are some diagrams which are $G$ invariant. Even for these diagrams it still simplifies to split the result into 2 parts which are related by the symmetry operation $\sigma$. In the following we will present the results of the rational parts from the various diagrams in a uniform way such that the complete result is obtained by summing over various individual terms and over the symmetry group, although the symmetry group $G$ only has rank 2 .

The 51 Feynman diagrams are split into 12 sets. We now give the results for the rational part from each set in turn, with some explanations of the tricks we used.

The first set shown in Fig. 15 has 4 Feynman diagrams. This includes the sole 6-point diagram. The logic for considering these four diagrams together is that the tensor reduction gives only two one-mass box diagrams which can

be easily computed following the method elucidated in [2]. The rational part is:

$$
\begin{aligned}
R_{1}(1) & =\frac{\langle 25\rangle^{2}\langle 56\rangle}{8\langle 26\rangle^{2}\langle 45\rangle}\left[1+\frac{s_{12}+s_{345}}{s_{12}-s_{345}}\right]\left(\epsilon_{1}, k_{6}\right)\left(\epsilon_{3}, k_{6}\right) \\
& +\frac{\langle 25\rangle\langle 56\rangle}{6\langle 26\rangle\langle 45\rangle}\left(\frac{\left(\epsilon_{1}, k_{6}\right)}{s_{12}-s_{345}}\left(\epsilon_{3}, k_{45}\right)\left(\epsilon_{6}, k_{12}\right)\right.
\end{aligned}
$$




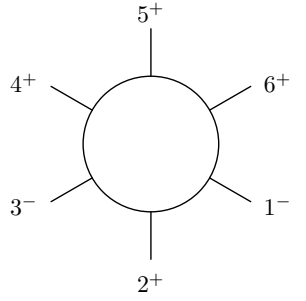

(a)

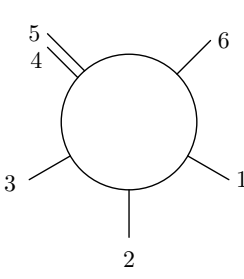

(b)

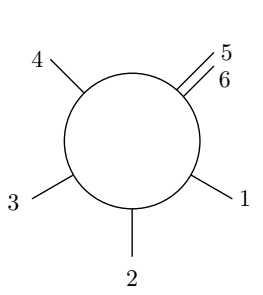

(c)

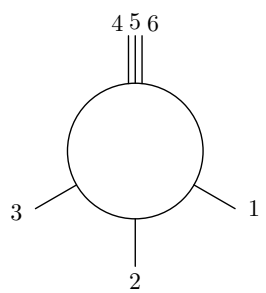

(d)

Figure 15: This set has 4 Feynman diagrams. See text for the logic of this combination.

$$
\begin{aligned}
& -\frac{1}{2} \frac{s_{12}+s_{345}}{\left(s_{12}-s_{345}\right)^{2}}\left(\epsilon_{3}, k_{6}\right)\left(\epsilon_{6}, k_{12}\right)\left(\epsilon_{1}, k_{6}\right) \\
& -\frac{1}{4} \frac{s_{12}+s_{345}}{s_{12}-s_{345}}\left(\left(\epsilon_{3}, k_{6}\right)\left(\epsilon_{6}, \epsilon_{1}\right)+\left(\epsilon_{1}, k_{6}\right)\left(\epsilon_{6}, \epsilon_{3}\right)\right) \\
& -\frac{1}{2}\left(\epsilon_{1}, \epsilon_{6}\right)\left(\epsilon_{3}, 2 k_{1}+k_{6}\right) \\
& \left.-\frac{1}{4}\left(\left(\epsilon_{1}, \epsilon_{6}\right)\left(\epsilon_{3}, k_{6}\right)+\left(\epsilon_{3}, \epsilon_{6}\right)\left(\epsilon_{1}, k_{6}\right)\right)\right)
\end{aligned}
$$

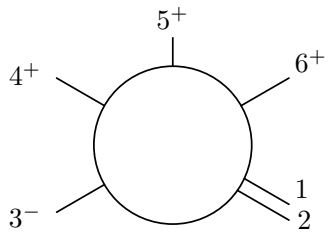

(a)

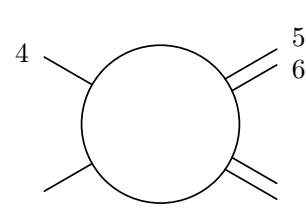

(b)

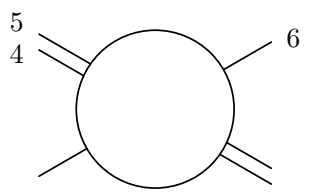

(c)

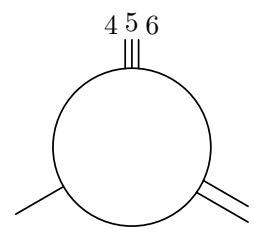

(d)

Figure 16: This set has 8 diagrams. The other 4 are obtained by the symmetry operation $\sigma$.

The 4 diagrams shown in Fig. 16 can be reduced similarly as in the above and the rational part is:

$$
\begin{aligned}
R_{2}(1)= & \frac{\langle 25\rangle}{6\langle 45\rangle}\left(\frac{\left(\epsilon_{3}, k_{45}\right)}{s_{12}-s_{345}}\left(\epsilon_{6}, k_{12}\right)\left(\epsilon_{12}, k_{6}\right)\right. \\
& -\frac{1}{2} \frac{s_{12}+s_{345}}{\left(s_{12}-s_{345}\right)^{2}}\left(\epsilon_{3}, k_{6}\right)\left(\epsilon_{6}, k_{12}\right)\left(\epsilon_{12}, k_{6}\right)
\end{aligned}
$$




$$
\begin{aligned}
& -\frac{1}{4} \frac{s_{12}+s_{345}}{s_{12}-s_{345}}\left(\left(\epsilon_{6}, \epsilon_{12}\right)\left(\epsilon_{3}, k_{6}\right)+\left(\epsilon_{6}, \epsilon_{3}\right)\left(\epsilon_{12}, k_{6}\right)\right) \\
& \left.-\frac{7}{12}\left(\left(\epsilon_{6}, \epsilon_{12}\right)\left(\epsilon_{3}, 2 k_{1}+k_{6}\right)+\left(\epsilon_{6}, \epsilon_{3}\right)\left(\epsilon_{12}, k_{6}\right)\right)\right) .
\end{aligned}
$$

by omitting a one-mass triangle diagram which will be cancelled by a bubble diagram from the tensor reduction of Fig. 19.

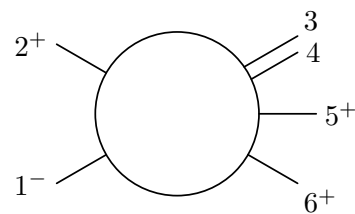

(a)

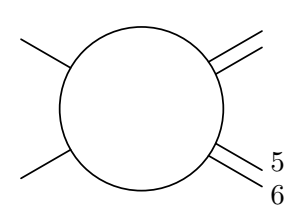

(b)

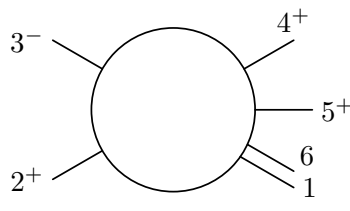

(c)

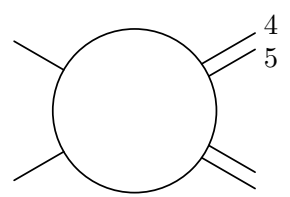

(d)

Figure 17: This set has 4 diagrams. The first 2 and the last 2 are related by symmetry operation $\sigma$.

The rational part from the diagrams in Fig. 17 is:

$$
\begin{aligned}
R_{3}(1)= & -\frac{\langle 25\rangle}{\langle 26\rangle}\left[-\frac{1}{4}\left(1+\frac{s_{12}+s_{345}}{s_{12}-s_{345}}\right)\left(\epsilon_{1}, k_{6}\right)\left(\epsilon_{34}, k_{6}\right)\right. \\
& \left.+\frac{1}{9}\left(5\left(k_{2}, k_{6}\right)\left(\epsilon_{34}, \epsilon_{1}\right)-4\left(\epsilon_{1}, k_{6}\right)\left(\epsilon_{34}, k_{2}\right)\right)\right] \\
+ & \frac{\langle 56\rangle}{3\langle 26\rangle}\left[\frac{\left(\epsilon_{34}, k_{5}\right)}{s_{12}-s_{345}}\left(\epsilon_{1}, k_{6}\right)\left(\tilde{\epsilon}_{6}, k_{1}\right)\right. \\
& -\frac{1}{2} \frac{s_{12}+s_{345}}{\left(s_{12}-s_{345}\right)^{2}}\left(\epsilon_{1}, k_{6}\right)\left(\epsilon_{34}, k_{6}\right)\left(\tilde{\epsilon}_{6}, k_{1}\right) \\
& -\frac{1}{4}\left(\frac{s_{12}+s_{345}}{s_{12}-s_{345}}+1\right)\left(\left(\epsilon_{34}, k_{6}\right)\left(\tilde{\epsilon}_{6}, \epsilon_{1}\right)+\left(\epsilon_{1}, k_{6}\right)\left(\tilde{\epsilon}_{6}, \epsilon_{34}\right)\right) \\
& \left.-\frac{1}{2}\left(\tilde{\epsilon}_{6}, \epsilon_{1}\right)\left(\epsilon_{34}, k_{1}-k_{5}\right)-\frac{2}{3}\left(\tilde{\epsilon}_{6}, \epsilon_{1}\right)\left(\epsilon_{34}, k_{2}\right)\right] \\
& \frac{\langle 56\rangle}{4\langle 26\rangle}\left(\epsilon_{3}, \epsilon_{4}\right)\left(\tilde{\epsilon}_{6}, \epsilon_{1}\right)\left(1+\frac{s_{61}}{s_{12}-s_{345}}\right) \\
& \frac{\langle 25\rangle}{12\langle 45\rangle}\left[\frac{2}{3}\left(\left(\epsilon_{2}, \epsilon_{61}\right)\left(\epsilon_{3}, k_{61}\right)+\left(\epsilon_{2}, k_{61}\right)\left(\epsilon_{3}, \epsilon_{61}\right)\right)\right. \\
& \left.+\frac{\left(\epsilon_{3}, k_{61}\right)}{s_{61}-s_{345}}\left(\epsilon_{2}, k_{61}\right)\left(\epsilon_{61}, k_{2}\right)\right]
\end{aligned}
$$


by also omitting a one-mass triangle diagram which will be cancelled by a bubble diagram from the tensor reduction of Fig. 19. Here $\tilde{\epsilon}_{6}=\lambda_{2} \tilde{\lambda}_{6}$.

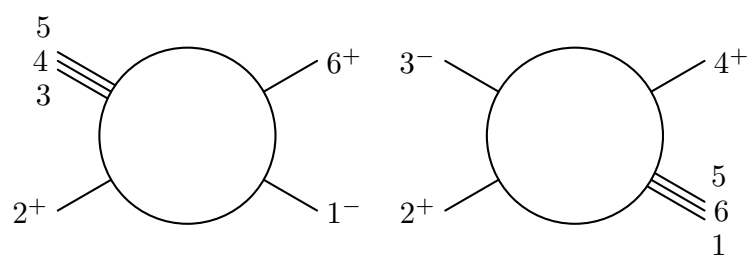

(a)

(b)

Figure 18: This set has 2 diagrams which are related by the symmetry operation $\sigma$.

The 4th set shown in Fig. 18 has 2 diagrams which are related by the symmetry operation $\sigma$. The rational part from the first diagram is:

$$
\begin{aligned}
R_{4}(1)= & -\frac{\langle 56\rangle}{4\langle 26\rangle}\left[\frac{\left(\epsilon_{1}, k_{6}\right)\left(\epsilon_{6}, k_{12}\right)}{s_{12}-s_{345}}+\left(\epsilon_{1}, \epsilon_{6}\right)\right]\left(\left(\epsilon_{3}, \epsilon_{45}\right)+\left(\epsilon_{34}, \epsilon_{5}\right)\right) \\
- & \frac{\langle 25\rangle\langle 56\rangle}{4\langle 26\rangle^{2}}\left[-\frac{s_{12}+s_{345}}{s_{12}-s_{345}}\left(\epsilon_{1}, k_{6}\right)\left(\epsilon_{345}, k_{6}\right)\right. \\
& \left.+\frac{8}{3}\left(k_{2}, k_{6}\right)\left(\epsilon_{1}, \epsilon_{345}\right)-\left(\epsilon_{1}, k_{6}\right)\left(\epsilon_{345}, 2 k_{2}+k_{6}\right)\right] \\
& +\frac{\langle 56\rangle}{3\langle 26\rangle}\left[-\frac{1}{2} \frac{s_{12}+s_{345}}{\left(s_{12}-s_{345}\right)^{2}}\left(\epsilon_{1}, k_{6}\right)\left(\epsilon_{6}, k_{12}\right)\left(\epsilon_{345}, k_{6}\right)\right. \\
& -\frac{1}{4}\left(\frac{s_{12}+s_{345}}{s_{12}-s_{345}}+1\right)\left(\left(\epsilon_{6}, \epsilon_{345}\right)\left(\epsilon_{1}, k_{6}\right)+\left(\epsilon_{6}, \epsilon_{1}\right)\left(\epsilon_{345}, k_{6}\right)\right) \\
& \left.-\frac{1}{2}\left(\epsilon_{6}, \epsilon_{1}\right)\left(\epsilon_{345}, k_{1}+2 k_{2}\right)+\frac{1}{3}\left(\left(\epsilon_{1}, \epsilon_{345}\right)\left(\epsilon_{6}, k_{2}\right)+\left(\epsilon_{6}, \epsilon_{1}\right)\left(\epsilon_{345}, k_{2}\right)\right)\right] \\
+ & \frac{\langle 25\rangle}{3\langle 26\rangle}\left[\frac{1}{3}\left(\left(\epsilon_{1}, \epsilon_{345}\right)\left(\epsilon_{2}, k_{6}\right)+\left(\epsilon_{2}, \epsilon_{345}\right)\left(\epsilon_{1}, k_{6}\right)\right)\right. \\
+ & \left.\frac{1}{2} \frac{\left(\epsilon_{345}, k_{2}\right)}{\left(s_{61}-s_{345}\right)}\left(\epsilon_{1}, k_{6}\right)\left(\epsilon_{2}, k_{61}\right)\right] .
\end{aligned}
$$

The rational part from the diagrams in Fig. 19 is:

$$
R_{5}(1)=-\frac{1}{6}\langle 25\rangle[56]\left(\epsilon_{12}, \epsilon_{34}\right)-\frac{\left(\tilde{\epsilon}_{6}, k_{1}\right)\left(\epsilon_{12}, k_{6}\right)}{12\left(s_{12}-s_{345}\right)}\left(3\left(\epsilon_{3}, \epsilon_{4}\right)-4\left(\epsilon_{34}, k_{5}\right)\right)
$$




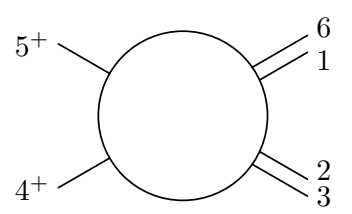

(a)

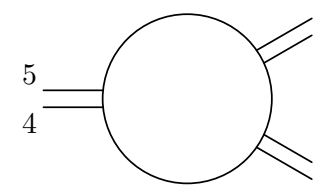

(b)

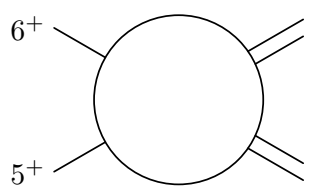

(c)

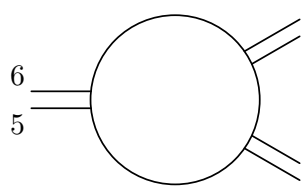

(d)

Figure 19: This set has 4 diagrams. The first 2 and the last 2 are related by symmetry operation $\sigma$.

$$
\begin{aligned}
& -\frac{1}{6} \frac{s_{12}+s_{345}}{\left(s_{12}-s_{345}\right)^{2}}\left(\tilde{\epsilon}_{6}, k_{1}\right)\left(\epsilon_{12}, k_{6}\right)\left(\epsilon_{34}, k_{6}\right) \\
& -\frac{1}{12} \frac{s_{12}+s_{345}}{s_{12}-s_{345}}\left(\left(\tilde{\epsilon}_{6}, \epsilon_{34}\right)\left(\epsilon_{12}, k_{6}\right)+\left(\tilde{\epsilon}_{6}, \epsilon_{12}\right)\left(\epsilon_{34}, k_{6}\right)\right) \\
& +\frac{1}{36}\left(-7\left(\tilde{\epsilon}_{6}, \epsilon_{34}\right)\left(\epsilon_{12}, k_{6}\right)+7\left(\tilde{\epsilon}_{6}, \epsilon_{12}\right)\left(\epsilon_{34}, 2 k_{5}+k_{6}\right)\right. \\
& \left.\quad+4\left(\tilde{\epsilon}_{6}, k_{1}\right)\left(\epsilon_{12}, \epsilon_{34}\right)-9\left(\tilde{\epsilon}_{6}, \epsilon_{12}\right)\left(\epsilon_{3}, \epsilon_{4}\right)\right),
\end{aligned}
$$

where $\tilde{\epsilon}_{6}=\lambda_{2} \tilde{\lambda}_{6}$ as defined before. As we said before, the bubble diagrams from tensor reduction cancel the rational part from the one-mass triangle diagrams from tensor reduction in Figs. 16 and 17.

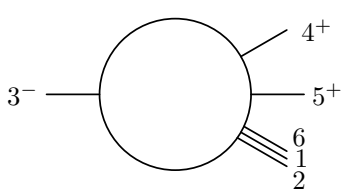

(a)

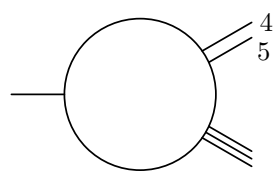

(b)

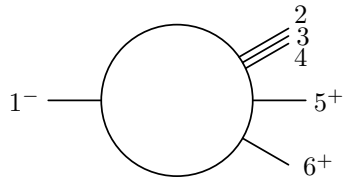

(c)

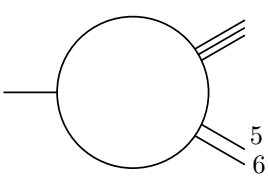

(d)

Figure 20: This set has 4 diagrams. The tensor reduction is by using the two adjacent same helicity legs $k_{4,5}$ for $(a)+(b)$, and $k_{5,6}$ for $(c)+(d)$.

The 4 diagrams shown in Fig. 20 can be reduced by using the two adjacent helicity legs $k_{4,5}$ or $k_{5,6}$ and the rational part from the first two diagrams is:

$$
\begin{aligned}
R_{6}(1)= & \frac{1}{9}\left(\left(\epsilon_{1}, \epsilon_{234}\right)\left(\tilde{\epsilon}_{6}, k_{1}\right)-\left(\tilde{\epsilon}_{6}, \epsilon_{234}\right)\left(\epsilon_{1}, k_{6}\right)+\frac{1}{2}\left(\epsilon_{234}, k_{6}-k_{1}\right)\left(\tilde{\epsilon}_{6}, \epsilon_{1}\right)\right) \\
& -\frac{\langle 25\rangle}{18\langle 45\rangle} s_{345}\left(\epsilon_{3}, \epsilon_{612}\right)-\frac{1}{6}\langle 25\rangle[56]\left(\epsilon_{1}, \epsilon_{234}\right) .
\end{aligned}
$$




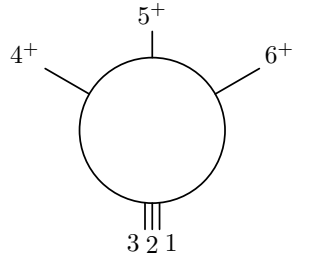

(a)

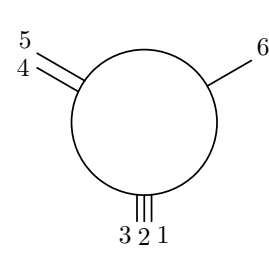

(b)

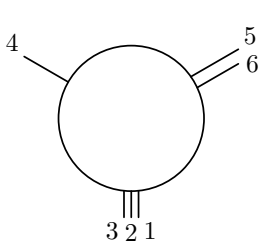

(c)

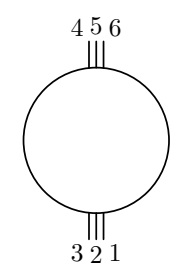

(d)

Figure 21: These diagrams have the same upper parts as the diagrams shown in Fig. 15. Here the bottom part has $k_{1,2,3}$ pinched together.

The 4 diagrams shown in Fig. 21 have the same upper parts as the 4 diagrams shown in Fig. 15 apart from the bottom parts which have $k_{1,2,3}$ pinched together. The rational part can be easily computed and we have:

$$
R_{7}(1)=-\frac{1}{6}\langle 25\rangle[56]\left(\epsilon_{4}, \epsilon_{123}\right)+\frac{1}{12}\langle 25\rangle\left[6\left|k_{123} \epsilon_{123}\right| 4\right] .
$$

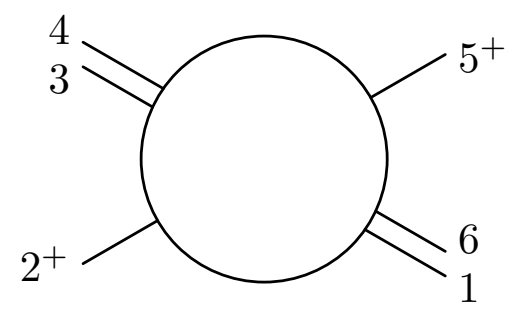

Figure 22: A two-mass-easy box diagram which is invariant under the symmetry operation $\sigma$.

The diagram shown in Fig. 22 is a two-mass-easy box diagram which is invariant under the symmetry operation $\sigma$. The rational part is:

$$
\begin{aligned}
R_{8}(1) & =-\frac{5}{18}\left(k_{2}, k_{5}\right)\left(\epsilon_{34}, \epsilon_{61}\right)-\frac{1}{4}\left(\epsilon_{34}, k_{5}\right)\left(\epsilon_{61}, k_{5}\right) \frac{s_{34}+s_{345}}{s_{34}-s_{345}} \\
& +\frac{4}{9}\left(\epsilon_{34}, k_{5}\right)\left(\epsilon_{61}, k_{2}\right)-\frac{1}{4}\left(\epsilon_{34}, k_{2}\right)\left(\epsilon_{61}, k_{2}\right) \frac{s_{61}+s_{345}}{s_{61}-s_{345}} .
\end{aligned}
$$

The rational part from the first diagram in Fig. 23 is:

$$
R_{9,1}(1)=\frac{1}{6} \frac{s_{61}+s_{345}}{\left(s_{61}-s_{345}\right)^{2}}\left(\epsilon_{2}, k_{61}\right)\left(\epsilon_{61}, k_{2}\right)\left(\epsilon_{345}, k_{2}\right)
$$




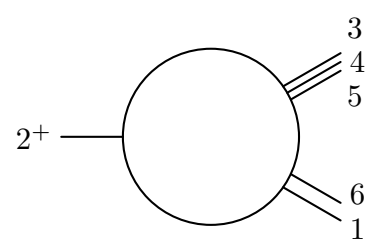

(a)

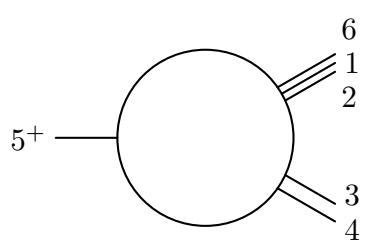

(b)

Figure 23: This set has 4 Feynman diagrams. The other 2 diagrams are obtained by symmetry operation $\sigma$ from the above 2 diagrams.

$$
\begin{aligned}
& +\frac{1}{12} \frac{s_{61}+s_{345}}{s_{61}-s_{345}}\left(\left(\epsilon_{2}, \epsilon_{345}\right)\left(\epsilon_{61}, k_{2}\right)+\left(\epsilon_{2}, \epsilon_{61}\right)\left(\epsilon_{345}, k_{2}\right)\right) \\
& -\frac{1}{4} \frac{\left(\epsilon_{2}, k_{61}\right)}{s_{61}-s_{345}}\left(\left(\epsilon_{61}, k_{2}\right)\left(\left(\epsilon_{3}, \epsilon_{45}\right)+\left(\epsilon_{34}, \epsilon_{5}\right)\right)+\left(\epsilon_{6}, \epsilon_{1}\right)\left(\epsilon_{345}, k_{2}\right)\right) \\
& -\frac{1}{36}\left(9\left(\left(\epsilon_{2}, \epsilon_{345}\right)\left(\epsilon_{6}, \epsilon_{1}\right)+\left(\epsilon_{2}, \epsilon_{61}\right)\left(\epsilon_{3}, \epsilon_{45}\right)+\left(\epsilon_{2}, \epsilon_{61}\right)\left(\epsilon_{34}, \epsilon_{5}\right)\right)\right. \\
& +7\left(-\left(\epsilon_{2}, \epsilon_{345}\right)\left(\epsilon_{61}, k_{2}\right)+\left(\epsilon_{2}, \epsilon_{61}\right)\left(\epsilon_{345}, k_{2}\right)\right) \\
& \left.+4\left(\epsilon_{61}, \epsilon_{345}\right)\left(\epsilon_{2}, k_{61}\right)\right)
\end{aligned}
$$

and the rational part from the 2nd diagram in Fig. 23 is:

$$
\begin{aligned}
R_{9,2}(1)= & \frac{1}{6} \frac{s_{34}+s_{345}}{\left(s_{34}-s_{345}\right)^{2}}\left(\epsilon_{5}, k_{34}\right)\left(\epsilon_{34}, k_{5}\right)\left(\epsilon_{612}, k_{5}\right) \\
& +\frac{1}{12} \frac{s_{34}+s_{345}}{s_{34}-s_{345}}\left(\left(\epsilon_{5}, \epsilon_{612}\right)\left(\epsilon_{34}, k_{5}\right)+\left(\epsilon_{5}, \epsilon_{34}\right)\left(\epsilon_{612}, k_{5}\right)\right) \\
& -\frac{1}{4} \frac{\left(\epsilon_{5}, k_{34}\right)}{s_{34}-s_{345}}\left(\left(\epsilon_{34}, k_{5}\right)\left(\left(\epsilon_{6}, \epsilon_{12}\right)+\left(\epsilon_{61}, \epsilon_{2}\right)\right)+\left(\epsilon_{3}, \epsilon_{4}\right)\left(\epsilon_{612}, k_{5}\right)\right) \\
& -\frac{1}{36}\left(9\left(\left(\epsilon_{5}, \epsilon_{612}\right)\left(\epsilon_{3}, \epsilon_{4}\right)+\left(\epsilon_{5}, \epsilon_{34}\right)\left(\left(\epsilon_{6}, \epsilon_{12}\right)+\left(\epsilon_{61}, \epsilon_{2}\right)\right)\right)\right. \\
& -7\left(\left(\epsilon_{5}, \epsilon_{612}\right)\left(\epsilon_{34}, k_{5}\right)-\left(\epsilon_{5}, \epsilon_{34}\right)\left(\epsilon_{612}, k_{5}\right)\right) \\
& \left.+4\left(\epsilon_{34}, \epsilon_{612}\right)\left(\epsilon_{5}, k_{34}\right)\right) .
\end{aligned}
$$

The rational part from the second diagram in Fig. 24 is:

$$
\begin{aligned}
R_{9,3}(1)= & -\frac{1}{6} \frac{s_{12}+s_{345}}{\left(s_{12}-s_{345}\right)^{2}}\left(\epsilon_{6}, k_{12}\right)\left(\epsilon_{12}, k_{6}\right)\left(\epsilon_{345}, k_{6}\right) \\
& -\frac{1}{12} \frac{s_{12}+s_{345}}{s_{12}-s_{345}}\left(\left(\epsilon_{6}, \epsilon_{345}\right)\left(\epsilon_{12}, k_{6}\right)+\left(\epsilon_{6}, \epsilon_{12}\right)\left(\epsilon_{345}, k_{6}\right)\right)
\end{aligned}
$$




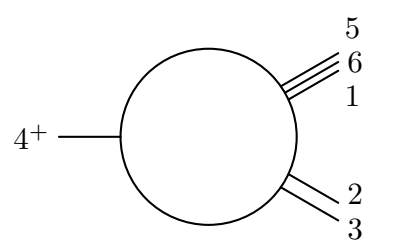

(a)

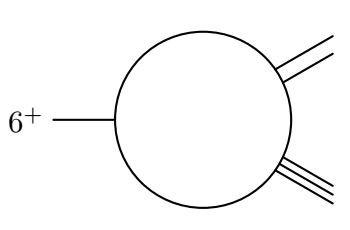

(b)

Figure 24: These 2 diagrams are related by the symmetry operation $\sigma$.

$$
\begin{aligned}
& -\frac{1}{4} \frac{\left(\epsilon_{6}, k_{12}\right)\left(\epsilon_{12}, k_{6}\right)}{s_{12}-s_{345}}\left(\left(\epsilon_{3}, \epsilon_{45}\right)+\left(\epsilon_{34}, \epsilon_{5}\right)\right) \\
& -\frac{1}{36}\left(9\left(\epsilon_{6}, \epsilon_{12}\right)\left(\left(\epsilon_{3}, \epsilon_{45}\right)+\left(\epsilon_{5}, \epsilon_{34}\right)\right)\right. \\
& +7\left(\left(\epsilon_{6}, \epsilon_{345}\right)\left(\epsilon_{12}, k_{6}\right)-\left(\epsilon_{6}, \epsilon_{12}\right)\left(\epsilon_{345}, k_{6}\right)\right) \\
& \left.-4\left(\epsilon_{12}, \epsilon_{345}\right)\left(\epsilon_{6}, k_{12}\right)\right) .
\end{aligned}
$$

Adding these three results together, we get:

$$
R_{9}(1)=R_{9,1}(1)+R_{9,2}(1)+R_{9,3}(1)
$$

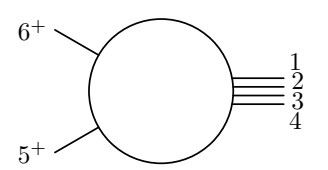

(a)

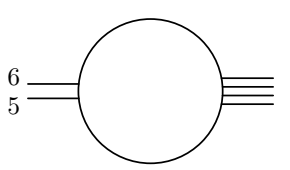

(b)

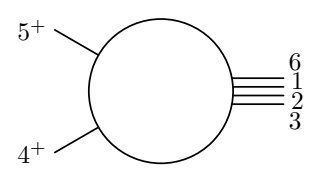

(c)

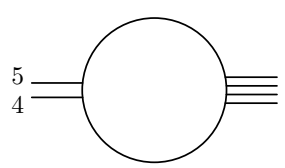

(d)

Figure 25: This set has 4 diagrams. Tensor reduction is easy with $k_{5,6}$ or $k_{4,5}$.

The rational part from the first 2 Feynman diagrams of fig. 25 is:

$$
\begin{aligned}
R_{10}(1) & =\frac{1}{6}\left(\epsilon_{5}, \epsilon_{6}\right)\left(\epsilon_{1234}, k_{5}\right) \\
& -\frac{1}{4}\left(\epsilon_{5}, \epsilon_{6}\right)\left(\left(\epsilon_{1}, \epsilon_{234}\right)+\left(\epsilon_{12}, \epsilon_{34}\right)+\left(\epsilon_{123}, \epsilon_{4}\right)\right) .
\end{aligned}
$$

There are other 8 Feynman diagrams which have the same form as given in Fig. 25, but with the 2 massless legs of the one-mass triangle having opposite 


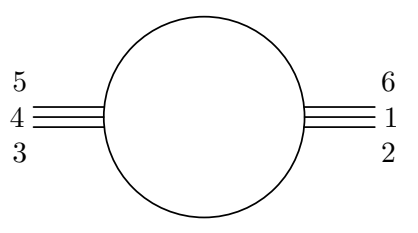

(a)

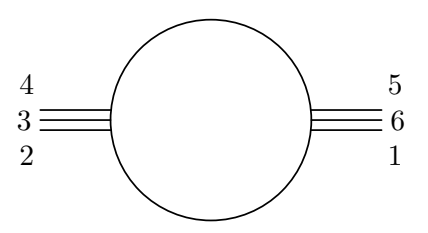

(b)

Figure 26: 2 bubble diagrams.

helicities. These give 0 by using the previous result, eq. (25). We will not show these diagrams here.

The last 2 diagrams are shown in Fig. 26 and the rational part is:

$$
R_{11}(1)=-\frac{1}{9} s_{345}\left(\epsilon_{345}, \epsilon_{612}\right) .
$$

Combining all the above results together, the complete rational part is given as follows:

$$
R=F \sum_{g \in G} R(g)=F \sum_{g \in G} \sum_{i=1}^{11} R_{i}(g),
$$

where

$$
F=-\frac{1}{[12][23]\langle 25\rangle^{2}\langle 45\rangle\langle 56\rangle},
$$

is an overall factor from the omitted factors of the polarization vectors.

The above result was also cross-checked with the explicit results of Berger, Bern, Dixon, Forder and Kosower [9] and we found exact (rational number) agreement.

\section{NMHV: $R\left(1^{-} 2^{-} 3^{+} 4^{-} 5^{+} 6^{+}\right)$}

Starting from this section we will compute the rational parts for the two non-MHV helicity configurations. These will give much more complicated algebraic expressions if one writes down the complete expressions. The strategy we use is to use another level of definition and express the result either explicitly or implicity through the (rational parts of the) 2-mass and 3-mass triangle integrals, and the 2-mass-hard box integrals. The explicit results in 
terms of the more elementary quantities, i.e., spinor products and kinematic variables, are collected in an appendix for quick reference.

For the $\operatorname{NMHV}\left(1^{-} 2^{-} 3^{+} 4^{-} 5^{+} 6^{+}\right)$, the symmetry group is $G=\{1, \sigma\}$ where $\sigma=\{i \leftrightarrow 7-i\}$ followed by conjugation. We can choose the following polarization vectors:

$$
\begin{aligned}
\epsilon_{1} & =\frac{\lambda_{1} \tilde{\lambda}_{2}}{[12]}, & \epsilon_{6} & =\frac{\lambda_{5} \tilde{\lambda}_{6}}{\langle 56\rangle}, \\
\epsilon_{2} & =\frac{\lambda_{2} \tilde{\lambda}_{1}}{[21]}, & \epsilon_{5} & =\frac{\lambda_{6} \tilde{\lambda}_{5}}{\langle 65\rangle}, \\
\epsilon_{4} & =\frac{\lambda_{4} \tilde{\lambda}_{2}}{[42]}, & \epsilon_{3} & =\frac{\lambda_{5} \tilde{\lambda}_{3}}{\langle 53\rangle} .
\end{aligned}
$$

We note again that we will omit the overall factors for all polarization vectors.

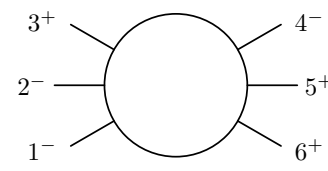

(a)

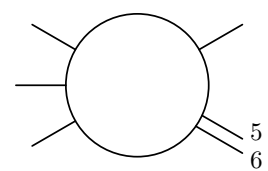

(b)

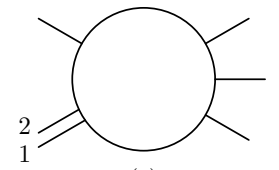

(c)

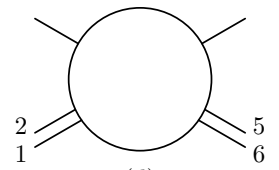

(d)

Figure 27: Diagrams (a) and (d) are invariant under the symmetry operation $\sigma$. Diagram (b) changes to diagram (c) under the symmetry operation $\sigma$.

The first set of diagrams are given in Fig. 27 which includes the only hexagon Feynman diagram. The tensor reduction can be done either with $k_{5,6}$ or $k_{1,2}$, but we can not do tensor reduction with both for all terms. By doing tensor reduction firstly with $k_{5,6}$, we have:

$$
\begin{aligned}
R_{1} & =\left(-I^{(1)} I^{(5)}+\frac{1}{2} I^{(5)} I^{(6)}+\frac{1}{2} I^{(1)} I^{(6)}\right)\left(\epsilon_{3}, p-k_{12}\right)\left(\epsilon_{4}, p-k_{123}\right) \\
& \times\left(\left(\epsilon_{1}, p\right)\left(\epsilon_{2}, p-k_{1}\right)+I^{(2)}\left(\left(\epsilon_{12}, p\right)+\frac{s_{12}}{2}\right)\right),
\end{aligned}
$$

where $I^{(i)}$ denotes the inverse propagator between $k_{i-1}$ and $k_{i}$. The last factor can be reduced further with the external momentum $k_{2}$ by noting the following relation:

$$
\left(\epsilon_{1}, p\right)\left(\epsilon_{2}, p-k_{1}\right)+I^{(2)}\left(\left(\epsilon_{12}, p\right)+\frac{s_{12}}{2}\right)=\frac{I^{(2)}}{2}\left(k_{12}, p-\frac{k_{12}}{2}\right)-I^{(3)}\left(k_{1}, p\right) .
$$


By using these results the rational part can be computed easily. We have:

$$
\begin{aligned}
R_{1} & =I_{3}^{3 m}\left(k_{1}, \epsilon_{3}, \epsilon_{4}\right)+\left(\epsilon_{4}, k_{5}\right) I_{3}^{3 m}\left(k_{1}, \epsilon_{3}\right) \\
& -\frac{1}{2}\left[\tilde{I}_{3}^{2 m(4)}\left(\epsilon_{4}, k_{1}, \epsilon_{3}\right)+\left(s_{56}-s_{234}\right) \tilde{I}_{3}^{2 m(4)}\left(\epsilon_{4}, \epsilon_{3}\right)\right] \\
& -\frac{1}{2}\left[I_{3}^{2 m(3)}\left(\epsilon_{3}, \epsilon_{4}, k_{12}\right)+s_{12} I_{3}^{2 m(3)}\left(\epsilon_{3}, \epsilon_{4}\right)\right] \\
& +\frac{1}{36} s_{34}\left(\epsilon_{34}, k_{12}\right)-\frac{1}{36}\left(\left(\epsilon_{3}, k_{2}\right)\left(\epsilon_{4}, k_{3}\right)-2 s_{23}\left(\epsilon_{3}, \epsilon_{4}\right)\right) \\
& -\frac{1}{72}\left(\left(\epsilon_{3}, k_{12}\right)\left(\epsilon_{4}, k_{56}\right)+2 s_{123}\left(\epsilon_{3}, \epsilon_{4}\right)\right) .
\end{aligned}
$$

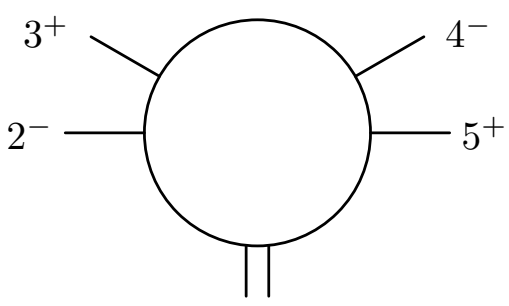

Figure 28: This diagram is invariant under the symmetry operation $\sigma: 1 \leftrightarrow$ $6,2 \leftrightarrow 5,3 \leftrightarrow 4$ (with conjugation).

The diagram shown in Fig. 28 is invariant under the symmetry operation $\sigma$. Its tensor reduction can be done for $k_{2}$ and $k_{5}$ because of our special choice of the reference momenta for $\epsilon_{3,4}$. We have:

$$
\begin{aligned}
R_{2}= & -\langle 43\rangle[32]\left(I^{(5)}-I^{(6)}\right) I^{(1)}\left(\lambda_{2} \tilde{\lambda}_{1}, p\right)\left(\lambda_{6} \tilde{\lambda}_{3}, p\right)\left(\epsilon_{61}, p\right) \\
+ & \langle 54\rangle[43]\left(I^{(2)}-I^{(3)}\right) I^{(1)}\left(\lambda_{4} \tilde{\lambda}_{1}, p\right)\left(\lambda_{6} \tilde{\lambda}_{5}, p\right)\left(\epsilon_{61}, p\right) \\
+ & \left(I^{(2)}\left(\lambda_{4} \tilde{\lambda}_{1}, p-k_{12}\right)-I^{(3)}\left(\lambda_{4} \tilde{\lambda}_{1}, p-k_{1}\right)\right) \\
& \times\left(I^{(5)}\left(\lambda_{6} \tilde{\lambda}_{3}, p+k_{6}\right)-I^{(6)}\left(\lambda_{6} \tilde{\lambda}_{3}, p+k_{56}\right)\right) I^{(1)} P_{61}(p),
\end{aligned}
$$

where

$$
P_{61}(p)=\left(\epsilon_{61}, p+k_{6}\right)-\frac{1}{2}\left(\epsilon_{6}, \epsilon_{1}\right) .
$$

The rational part is computed by using the formulas for box and triangle integrals and we have:

$$
R_{2}=-\langle 43\rangle[32]\left(I_{4}^{2 m h(2)}\left(\lambda_{2} \tilde{\lambda}_{1}, \lambda_{6} \tilde{\lambda}_{3}, \epsilon_{61}\right)-I_{4}^{1 m(2)}\left(\lambda_{2} \tilde{\lambda}_{1}, \lambda_{6} \tilde{\lambda}_{3}, \epsilon_{61}\right)\right)
$$




$$
\begin{aligned}
& +\langle 54\rangle[43]\left(I_{4}^{1 m(3)}\left(\lambda_{4} \tilde{\lambda}_{1}, \lambda_{6} \tilde{\lambda}_{5}, \epsilon_{61}\right)-I_{4}^{2 m h(4)}\left(\lambda_{4} \tilde{\lambda}_{1}, \lambda_{6} \tilde{\lambda}_{5}, \epsilon_{61}\right)\right) \\
& +I_{3}^{2 m(3)}\left(\lambda_{6} \tilde{\lambda}_{3}, \epsilon_{61}, \lambda_{4} \tilde{\lambda}_{1}\right)-\left\langle 6\left|k_{45}\right| 3\right] I_{3}^{2 m(3)}\left(\lambda_{4} \tilde{\lambda}_{1}, \epsilon_{61}\right) \\
& -\quad\left(\left(\epsilon_{61}, k_{45}\right)+\frac{1}{2}\left(\epsilon_{6}, \epsilon_{1}\right)\right) I_{3}^{2 m(3)}\left(\lambda_{6} \tilde{\lambda}_{3}, \lambda_{4} \tilde{\lambda}_{1}\right) \\
& +\tilde{I}_{3}^{2 m(4)}\left(\lambda_{4} \tilde{\lambda}_{1}, \lambda_{6} \tilde{\lambda}_{3}, \epsilon_{61}\right)+\left\langle 4\left|k_{23}\right| 1\right] \tilde{I}_{3}^{2 m(4)}\left(\lambda_{6} \tilde{\lambda}_{3}, \epsilon_{61}\right) \\
& +\quad\left(\left(\epsilon_{61}, k_{23}\right)-\frac{1}{2}\left(\epsilon_{6}, \epsilon_{1}\right)\right) \tilde{I}_{3}^{2 m(4)}\left(\lambda_{6} \tilde{\lambda}_{3}, \lambda_{4} \tilde{\lambda}_{1}\right) \\
& -I_{3}^{3 m}\left(\lambda_{4} \tilde{\lambda}_{1}, \epsilon_{61}, \lambda_{6} \tilde{\lambda}_{3}\right)-\left(\left(\epsilon_{61}, k_{23}\right)-\frac{1}{2}\left(\epsilon_{6}, \epsilon_{1}\right)\right) I_{3}^{3 m}\left(\lambda_{4} \tilde{\lambda}_{1}, \lambda_{6} \tilde{\lambda}_{3}\right) \\
& +\frac{7}{18} s_{34}\left(\tilde{\epsilon}_{34}, \epsilon_{61}\right)+\frac{1}{4}\langle 64\rangle[13]\left(\epsilon_{61}, k_{3}-k_{4}\right),
\end{aligned}
$$

where

$$
\begin{aligned}
\tilde{\epsilon}_{34} & =\left.\epsilon_{34}\right|_{\epsilon_{3} \rightarrow \lambda_{6} \tilde{\lambda}_{3}, \epsilon_{4} \rightarrow \lambda_{4} \tilde{\lambda}_{1}} \\
& =\frac{\langle 64\rangle}{\langle 34\rangle} \lambda_{4} \tilde{\lambda}_{1}-\frac{[13]}{[43]} \lambda_{6} \tilde{\lambda}_{3}+\frac{\langle 64\rangle[13]}{2\langle 34\rangle[43]}\left(k_{3}-k_{4}\right)
\end{aligned}
$$

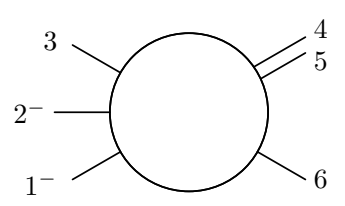

(a)

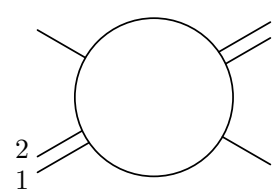

(b)

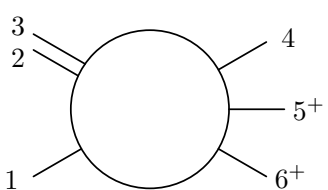

(c)

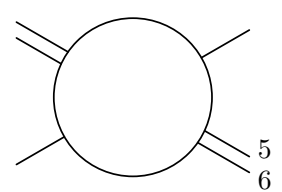

(d)

Figure 29: 4 Feynman diagrams which can be reduced easily with $k_{1,2}$ ((a) and $(\mathrm{b}))$ or $k_{5,6}((\mathrm{c})$ and $(\mathrm{d}))$. (c) and (d) are obtained from (a) and (b) by symmetry operation $\sigma$.

The four Feynman diagrams in Fig. 29 are related by the symmetry operation $\sigma$. The rational part from diagrams (a) and (b) is:

$$
\begin{aligned}
R_{3}(1) & =\frac{1}{2} I_{3}^{2 m(3)}\left(\epsilon_{3}, \epsilon_{45}, \epsilon_{6}\right)+\frac{1}{4} I_{3}^{2 m(3)}\left(\epsilon_{3}, 2\left(\epsilon_{6}, k_{12}\right) \epsilon_{45}-\left(\epsilon_{4}, \epsilon_{5}\right) \epsilon_{6}\right) \\
& +\frac{1}{2} \tilde{I}_{3}^{2 m(6)}\left(\epsilon_{6}, \epsilon_{3}, \epsilon_{45}\right)-\frac{1}{4} \tilde{I}_{3}^{2 m(6)}\left(\epsilon_{6}, 2\left(\epsilon_{3}, k_{12}\right) \epsilon_{45}+\left(\epsilon_{4}, \epsilon_{5}\right) \epsilon_{3}\right) \\
& -I_{3}^{3 m}\left(\epsilon_{45}, \epsilon_{3}, \epsilon_{6}\right)+\left(\left(\epsilon_{45}, k_{23}\right)+\frac{1}{2}\left(\epsilon_{4}, \epsilon_{5}\right)\right) I_{3}^{3 m}\left(\epsilon_{3}, \epsilon_{6}\right) .
\end{aligned}
$$




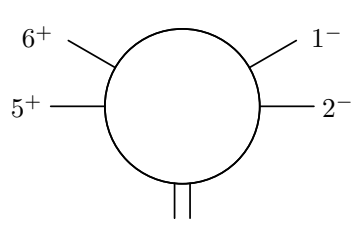

(a)

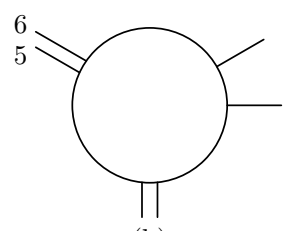

(b)

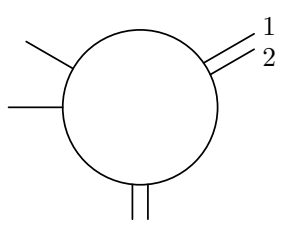

(c)

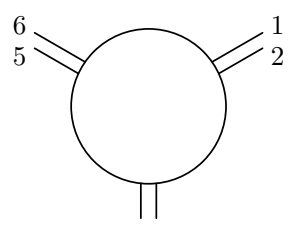

(d)

Figure 30: These 4 diagrams have the same structure as those in Fig. 27 with $k_{3,4}$ replaced by $k_{34}$.

The rational part from diagrams (c) and (d) is denoted as $R_{3}(\sigma)$. It is obtained from $R_{3}(1)$ by the symmetry operation $\sigma$.

For the 4 diagrams shown in Fig. 30, the rational part can be obtained by using the same tensor reduction techniques as for Fig. 27 and the rational part is:

$$
\begin{aligned}
R_{4} & =\frac{1}{36}\left(\left(\epsilon_{34}, k_{2}\right)\left(k_{1}, k_{56}\right)-\left(\epsilon_{34}, k_{5}\right)\left(k_{6}, k_{12}\right)\right) \\
& +\frac{1}{18}\left(\left(\epsilon_{34}, k_{1}\right)\left(k_{5}, k_{61}\right)-\left(\epsilon_{34}, k_{6}\right)\left(k_{2}, k_{61}\right)\right) \\
& -\frac{1}{72} s_{34}\left(\epsilon_{34}, k_{12}-k_{56}\right)-\frac{1}{6}\left(s_{12}\left(\epsilon_{34}, \epsilon_{56}\right)+s_{56}\left(\epsilon_{34}, \epsilon_{12}\right)\right) .
\end{aligned}
$$

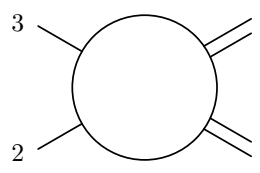

(a)

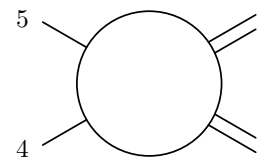

(b)

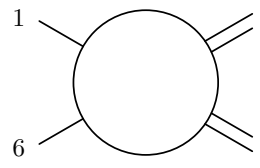

(c)

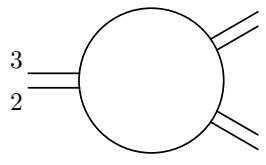

(d)

Figure 31: Four diagrams which should be computed individually.

The rational part for the 3 two-mass-hard box diagrams and the threemass triangle diagram is:

$$
\begin{aligned}
R_{5} & =I_{4}^{2 m h(2)}\left(\lambda_{2} \tilde{\lambda}_{1}, \lambda_{5} \tilde{\lambda}_{3}, \epsilon_{45}, \epsilon_{61} ;-\left(\epsilon_{4}, \epsilon_{5}\right) / 2,-\left(\epsilon_{6}, \epsilon_{1}\right) / 2\right) \\
& +I_{4}^{2 m h(4)}\left(\lambda_{4} \tilde{\lambda}_{2}, \lambda_{6} \tilde{\lambda}_{5}, \epsilon_{61}, \epsilon_{23} ;-\left(\epsilon_{6}, \epsilon_{1}\right) / 2,-\left(\epsilon_{2}, \epsilon_{3}\right) / 2\right) \\
& +I_{4}^{2 m h(6)}\left(\lambda_{5} \tilde{\lambda}_{6}, \lambda_{1} \tilde{\lambda}_{2}, \epsilon_{23}, \epsilon_{45} ;-\left(\epsilon_{2}, \epsilon_{3}\right) / 2,-\left(\epsilon_{4}, \epsilon_{5}\right) / 2\right) \\
& +I_{3}^{3 m}\left(\epsilon_{23}, \epsilon_{45}, \epsilon_{61}\right)-\frac{1}{2}\left(\epsilon_{2}, \epsilon_{3}\right) I_{3}^{3 m}\left(\epsilon_{45}, \epsilon_{61}\right)
\end{aligned}
$$




$$
-\frac{1}{2}\left(\epsilon_{4}, \epsilon_{5}\right) I_{3}^{3 m}\left(\epsilon_{61}, \epsilon_{23}\right)-\frac{1}{2}\left(\epsilon_{6}, \epsilon_{1}\right) I_{3}^{3 m}\left(\epsilon_{23}, \epsilon_{45}\right),
$$

just by using the definition of $I_{4}^{2 m h(i)}$ and $I_{3}^{3 m}$. See the appendix for explicit formulas.

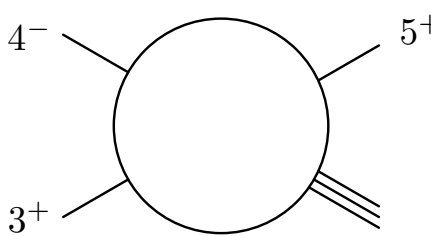

(a)

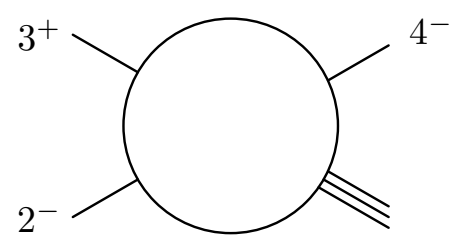

(b)

Figure 32: 2 Feynman diagrams. The 2nd can be obtained from the first one by the symmetry operation $\sigma$.

The first diagram in Fig. 32 gives the following rational part:

$$
\begin{aligned}
R_{6}(1)= & \frac{\langle 65\rangle}{\langle 35\rangle}\left[\frac{4}{9}\left(\left(\epsilon_{4}, k_{3}\right)\left(\epsilon_{612}, k_{5}\right)+\left(\epsilon_{4}, k_{5}\right)\left(\epsilon_{612}, k_{3}\right)\right)\right. \\
& -\frac{5}{9}\left(k_{3}, k_{5}\right)\left(\epsilon_{4}, \epsilon_{612}\right)+\frac{1}{4}\left(\left(\epsilon_{4}, k_{3}\right)\left(\epsilon_{612}, k_{3}\right)+\left(\epsilon_{4}, k_{5}\right)\left(\epsilon_{612}, k_{5}\right)\right) \\
& \left.+\frac{1}{4}\left[\frac{s_{45}+s_{345}}{s_{45}-s_{345}}\left(\epsilon_{4}, k_{3}\right)\left(\epsilon_{612}, k_{3}\right)+\frac{s_{34}+s_{345}}{s_{34}-s_{345}}\left(\epsilon_{4}, k_{5}\right)\left(\epsilon_{612}, k_{5}\right)\right]\right] \\
+ & \frac{\langle 63\rangle}{\langle 53\rangle}\left[-\frac{1}{9} s_{34}\left(\epsilon_{34}, \epsilon_{612}\right)+I_{3}^{2 m(3)}\left(\epsilon_{3}, \epsilon_{4}, \epsilon_{612}\right)\right. \\
& \left.-\frac{1}{2}\left(\left(\epsilon_{6}, \epsilon_{12}\right)+\left(\epsilon_{61}, \epsilon_{2}\right)\right) I_{3}^{2 m(3)}\left(\epsilon_{3}, \epsilon_{4}\right)\right] .
\end{aligned}
$$

The rational part of the other diagram Fig. 32 is $R_{6}(\sigma)$ and it is obtained from $R_{6}(1)$ by the symmetry operation $\sigma$.

The four diagrams shown in Fig. 33 give the following rational part:

$$
\begin{aligned}
R_{7}(1) & =\frac{1}{18}\left(\epsilon_{4}, k_{5}\right)\left(\epsilon_{123}, k_{6}\right)+\frac{1}{18}\left(2 s_{45}-s_{123}-3 s_{56}\right)\left(\epsilon_{123}, \epsilon_{4}\right) \\
& +\frac{1}{18}\left(\epsilon_{1}, k_{6}\right)\left(\epsilon_{234}, k_{5}\right)+\frac{1}{18}\left(2 s_{61}-s_{234}-3 s_{56}\right)\left(\epsilon_{1}, \epsilon_{234}\right) .
\end{aligned}
$$

$R_{7}(\sigma)$ is obtained from $R_{7}(1)$ by the symmetry operation $\sigma$ (with conjugation). 


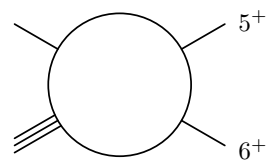

(a)

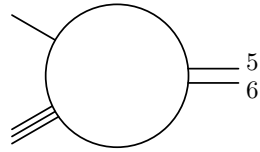

(b)

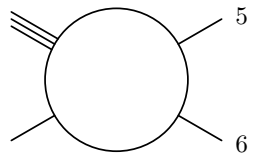

(c)

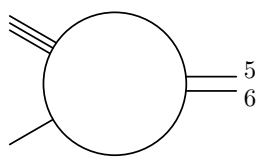

(d)

Figure 33: This set has 8 Feynman diagrams. Four more diagrams are obtained from these four diagrams by the symmetry operation $\sigma$. The above four diagrams can be reduced easily with $k_{5,6}$.

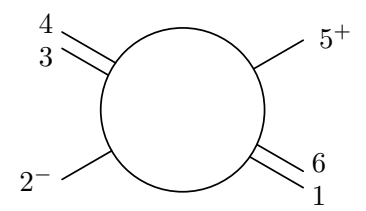

Figure 34: A two-mass-easy box diagram.

The rational part from the diagram shown in Fig. 34 decomposes as $R_{8}(1)+R_{8}(\sigma)$ and we have:

$$
\begin{aligned}
R_{8}(1)= & \frac{[12]\langle 65\rangle}{[52]\langle 25\rangle}\left[\frac{5}{18}\left\langle 2\left|\epsilon_{34}\right| 5\right]\left\langle 2\left|\epsilon_{61}\right| 5\right]+\frac{\left\langle 2\left|k_{34}\right| 5\right]^{2}\left(\epsilon_{34}, k_{2}\right)\left(\epsilon_{61}, k_{2}\right)}{3\left(s_{34}-s_{234}\right)\left(s_{61}-s_{345}\right)}\right] \\
+ & \frac{\langle 62\rangle}{\langle 52\rangle}\left[\tilde{I}_{3}^{2 m(2)}\left(\lambda_{2} \tilde{\lambda}_{1}, \epsilon_{34}, \epsilon_{61}\right)-\frac{1}{2} \tilde{I}_{3}^{2 m(2)}\left(\lambda_{2} \tilde{\lambda}_{1},\left(\epsilon_{6}, \epsilon_{1}\right) \epsilon_{34}+\left(\epsilon_{3}, \epsilon_{4}\right) \epsilon_{61}\right)\right. \\
& \left.-I_{3}^{2 m(2)}\left(\lambda_{2} \tilde{\lambda}_{1}, \epsilon_{34}, \epsilon_{61}\right)+\frac{1}{2} I_{3}^{2 m(2)}\left(\lambda_{2} \tilde{\lambda}_{1},\left(\epsilon_{6}, \epsilon_{1}\right) \epsilon_{34}+\left(\epsilon_{3}, \epsilon_{4}\right) \epsilon_{61}\right)\right] \\
- & \frac{[15]\langle 62\rangle}{18[25]\langle 52\rangle}\left(\left(k_{2}, k_{5}\right)\left(\epsilon_{34}, \epsilon_{61}\right)-\left(\epsilon_{34}, k_{2}\right)\left(\epsilon_{61}, k_{5}\right)\right),
\end{aligned}
$$

whereas $R_{8}(\sigma)$ is obtained from $R_{8}(1)$ by the symmetry operation $\sigma$ (with conjugation).

The diagrams in Fig. 35 give the following rational part:

$$
\begin{aligned}
R_{9}(1) & =I_{3}^{2 m(1)}\left(\epsilon_{1}, \epsilon_{23}, \epsilon_{456}\right)+I_{3}^{2 m(3)}\left(\epsilon_{3}, \epsilon_{45}, \epsilon_{612}\right) \\
& +I_{3}^{2 m(2)}\left(\epsilon_{2}, \epsilon_{34}, \epsilon_{561}\right)+\tilde{I}_{3}^{2 m(2)}\left(\epsilon_{2}, \epsilon_{345}, \epsilon_{61}\right) \\
& -\frac{1}{2} I_{3}^{2 m(1)}\left(\epsilon_{1},\left(\epsilon_{2}, \epsilon_{3}\right) \epsilon_{456}+\left(\left(\epsilon_{4}, \epsilon_{56}\right)+\left(\epsilon_{45}, \epsilon_{6}\right)\right) \epsilon_{23}\right) \\
& -\frac{1}{2} I_{3}^{2 m(3)}\left(\epsilon_{3},\left(\epsilon_{4}, \epsilon_{5}\right) \epsilon_{612}+\left(\left(\epsilon_{6}, \epsilon_{12}\right)+\left(\epsilon_{61}, \epsilon_{2}\right)\right) \epsilon_{45}\right)
\end{aligned}
$$




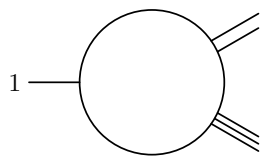

(a)

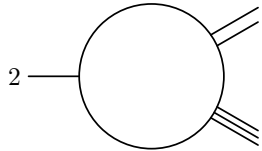

(b)

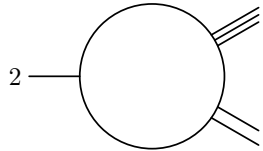

(c)

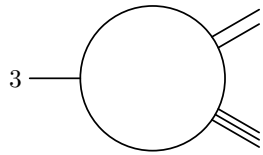

(d)

Figure 35: Four 2-mass triangle diagrams which should be computed individually.

$$
\begin{aligned}
& -\frac{1}{2} I_{3}^{2 m(2)}\left(\epsilon_{2},\left(\epsilon_{3}, \epsilon_{4}\right) \epsilon_{561}+\left(\left(\epsilon_{5}, \epsilon_{61}\right)+\left(\epsilon_{56}, \epsilon_{1}\right)\right) \epsilon_{34}\right) \\
& -\frac{1}{2} \tilde{I}_{3}^{2 m(2)}\left(\epsilon_{2},\left(\left(\epsilon_{3}, \epsilon_{45}\right)+\left(\epsilon_{34}, \epsilon_{5}\right)\right) \epsilon_{61}+\left(\epsilon_{6}, \epsilon_{1}\right) \epsilon_{345}\right) .
\end{aligned}
$$

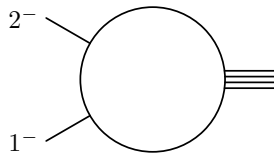

(a)

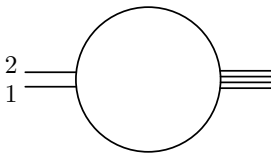

(b)

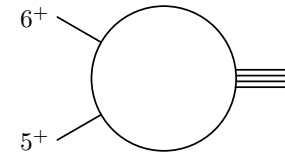

(c)

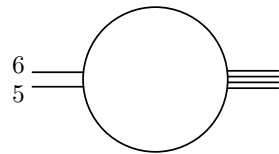

(d)

Figure 36: Four 1-mass triangle diagrams which can be computed either directly or by tensor reduction with $k_{1,2}$ or $k_{5,6}$.

The rational part from the first two diagrams in Fig. 36 is:

$$
R_{10}(1)=\frac{1}{4} s_{12}\left(\left(\epsilon_{3}, \epsilon_{456}\right)+\left(\epsilon_{34}, \epsilon_{56}\right)+\left(\epsilon_{3}, \epsilon_{456}\right)\right)-\frac{1}{6} s_{12}\left(\epsilon_{3456}, k_{1}\right) .
$$

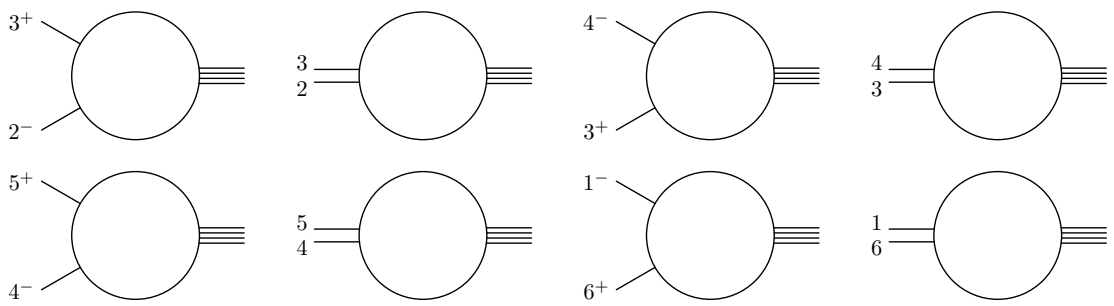

Figure 37: These 8 diagrams give a vanishing contribution to the rational part. 


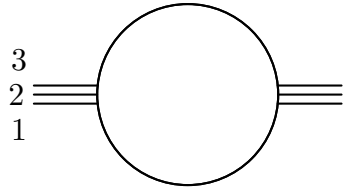

(a)

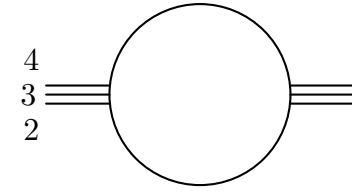

(b)

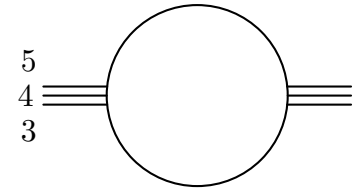

(c)

Figure 38: The three remaining bubble diagrams.

The last contribution to the rational part is from the remaining three bubble diagrams shown in Fig. 38 and it is given by:

$$
R_{11}=-\frac{1}{9}\left(s_{123}\left(\epsilon_{123}, \epsilon_{456}\right)+s_{234}\left(\epsilon_{234}, \epsilon_{561}\right)+s_{345}\left(\epsilon_{345}, \epsilon_{612}\right)\right) .
$$

The complete result for the rational part is:

$$
R=F\left[\sum_{i=1,2,4,5,11} R_{i}+\sum_{i=3,6,7,8,9,10} \sum_{g} R_{i}(g)\right]
$$

where

$$
F=\frac{1}{\langle 35\rangle[24]([12]\langle 56\rangle)^{2}},
$$

is an overall factor.

\section{$7 \quad$ NMHV: $R\left(1^{-} 2^{+} 3^{-} 4^{+} 5^{-} 6^{+}\right)$}

In this case we choose the following polarization vectors:

$$
\begin{array}{lll}
\epsilon_{1}=\frac{\lambda_{1} \tilde{\lambda}_{5}}{[15]}, & \epsilon_{2}=\frac{\lambda_{6} \tilde{\lambda}_{2}}{\langle 62\rangle}, \\
\epsilon_{3}=\frac{\lambda_{3} \tilde{\lambda}_{1}}{[31]}, & \epsilon_{4}=\frac{\lambda_{2} \tilde{\lambda}_{4}}{\langle 24\rangle}, \\
\epsilon_{5}=\frac{\lambda_{5} \tilde{\lambda}_{3}}{[53]}, & \epsilon_{6}=\frac{\lambda_{4} \tilde{\lambda}_{6}}{\langle 46\rangle} .
\end{array}
$$

This helicity configuration has the biggest symmetry group which is $Z_{6}$ generated by $\tau: i \rightarrow i+1$ accompanied by conjugation. Because of this symmetry 
group, we need only to compute a few diagrams from each set and the complete result will be obtained by a summation over the symmetry group. For an invariant diagram, tensor reduction often (partially) destroys the symmetry and the symmetry is not manifest in the resulting expression of the rational part. In this case it is often simpler to give the complete result for this invariant diagram as a whole or as a summation over only a subgroup of the symmetry group $Z_{6}$.

Let us first start with the diagram (a) in Fig. 2. We can do the tensor reduction with $k_{1,4}$ and we have

$$
\begin{aligned}
R_{1} & =-\langle 45\rangle[56]\left(I^{(1)}-I^{(2)}\right)\left(I^{(3)}-I^{(4)}\right)\left(I^{(5)}-I^{(6)}\right)\left(\lambda_{2} \tilde{\lambda}_{4}, p\right)\left(\lambda_{6} \tilde{\lambda}_{2}, p\right) \\
& -\langle 32\rangle[21]\left(I^{(2)}-I^{(3)}\right)\left(I^{(4)}-I^{(5)}\right)\left(I^{(6)}-I^{(1)}\right)\left(\lambda_{5} \tilde{\lambda}_{3}, p\right)\left(\lambda_{1} \tilde{\lambda}_{5}, p\right) \\
& -I^{(1)} I^{(5)}\left(\lambda_{3} \tilde{\lambda}_{5}, p-k_{1}\right)\left(\lambda_{6} \tilde{\lambda}_{2}, p-k_{12}\right)\left(\lambda_{2} \tilde{\lambda}_{6}, p-k_{123}\right)\left(\lambda_{5} \tilde{\lambda}_{3}, p+k_{6}\right) \\
& -I^{(2)} I^{(4)}\left(\lambda_{2} \tilde{\lambda}_{6}, p+k_{56}\right)\left(\lambda_{5} \tilde{\lambda}_{3}, p+k_{6}\right)\left(\lambda_{3} \tilde{\lambda}_{5}, p\right)\left(\lambda_{6} \tilde{\lambda}_{2}, p-k_{12}\right) \\
& +I^{(1)} I^{(4)}\left(\lambda_{3} \tilde{\lambda}_{5}, p-k_{1}\right)\left(\lambda_{6} \tilde{\lambda}_{2}, p-k_{12}\right)\left(\lambda_{2} \tilde{\lambda}_{6}, p+k_{56}\right)\left(\lambda_{5} \tilde{\lambda}_{3}, p+k_{6}\right) \\
& +I^{(2)} I^{(5)}\left(\lambda_{6} \tilde{\lambda}_{2}, p-k_{12}\right)\left(\lambda_{2} \tilde{\lambda}_{6}, p-k_{123}\right)\left(\lambda_{5} \tilde{\lambda}_{3}, p+k_{6}\right)\left(\lambda_{3} \tilde{\lambda}_{5}, p\right) . \quad(77)
\end{aligned}
$$

We denote the contribution (to the rational part) of each term by $R_{1}^{(i)}, i=$ $1, \cdots, 6$. Let us compute the various terms explicitly. We have

$$
\begin{aligned}
R_{1}^{(1)}= & -\langle 45\rangle[56]\left[\frac{1}{2}\left[\frac{\left\langle 2\left|k_{56}\right| 4\right]}{s_{56}-s_{456}}-\frac{\left\langle 2\left|k_{23}\right| 4\right]}{s_{23}-s_{234}}\right]\left(\lambda_{6} \tilde{\lambda}_{2}, k_{4}\right)\right. \\
& +I_{3}^{3 m}\left(\lambda_{2} \tilde{\lambda}_{4}, \lambda_{6} \tilde{\lambda}_{2}\right)-\tilde{I}_{3}^{3 m}\left(\lambda_{2} \tilde{\lambda}_{4}, \lambda_{6} \tilde{\lambda}_{2}\right) \\
& \left.+\frac{1}{2}\left[\frac{\left\langle 6\left|k_{12}\right| 2\right]}{s_{12}-s_{345}}-\frac{\left\langle 6\left|k_{45}\right| 2\right]}{s_{45}-s_{123}}\right]\left(\lambda_{2} \tilde{\lambda}_{4}, k_{6}\right)\right] .
\end{aligned}
$$

$R_{1}^{(2)}$ can be obtained from $R_{1}^{(1)}$ by the symmetry operation $\tau^{3}$, i.e.:

$$
R_{1}^{(2)}=\left(\left.R_{1}^{(1)}\right|_{i \rightarrow i+3}\right)^{*},
$$

where $*$ denotes conjugation: \langle\rangle$\leftrightarrow[]$.

The contribution from the 3rd term in eq. (77) is:

$$
\begin{aligned}
R_{1}^{(3)} & =-\frac{1}{6}\left(k_{2}, k_{6}\right)\left(k_{3}, k_{5}\right)-\left\langle 3\left|k_{2}\right| 5\right] I_{3}^{2 m(3)}\left(\lambda_{5} \tilde{\lambda}_{3}, k_{6}\right) \\
& +\left\langle 5\left|k_{4}\right| 3\right] I_{3}^{2 m(3)}\left(\lambda_{3} \tilde{\lambda}_{5}, k_{6}\right)-I_{3}^{2 m(3)}\left(\lambda_{3} \tilde{\lambda}_{5}, \lambda_{5} \tilde{\lambda}_{3}, k_{6}\right) \\
& +\left(k_{6}, k_{23}\right) I_{3}^{3 m}\left(\lambda_{3} \tilde{\lambda}_{5}, \lambda_{5} \tilde{\lambda}_{3}\right)+I_{3}^{3 m}\left(\lambda_{3} \tilde{\lambda}_{5}, k_{6}, \lambda_{5} \tilde{\lambda}_{3}\right) \\
& +\left\langle 2\left|k_{3}\right| 6\right]\left[I_{3}^{3 m}\left(\lambda_{6} \tilde{\lambda}_{2}, k_{5}\right)-\frac{1}{2}\left[\left\langle 6\left|k_{5}\right| 2\right]+\frac{\left\langle 6\left|k_{1}\right| 2\right]\left(k_{2}, k_{5}\right)}{s_{61}-s_{345}}\right]\right] .
\end{aligned}
$$


$R_{1}^{(4)}$ can be obtained from $R_{1}^{(3)}$ by the symmetry operation $\tau^{3}$ just as for the term $R_{1}^{(2)}$ from $R_{1}^{(1)}$.

The rational parts from the last two terms in eq. (77) are

$$
\begin{aligned}
R_{1}^{(5)}= & \frac{1}{2}\left(k_{3}, k_{5}\right)\left(k_{2}, k_{6}\right)+\frac{1}{18}\left(2\left(k_{3}, k_{6}\right)\left(k_{2}, k_{5}\right)\right. \\
& \left.-\left(k_{5}, k_{3}\right)\left(k_{2}, k_{6}\right)-\left(k_{5}, k_{6}\right)\left(k_{2}, k_{3}\right)\right) \\
- & \frac{1}{2}\left[\frac{\left\langle 2\left|k_{34} k_{6} k_{61}\right| 2\right]}{s_{61}-s_{345}}-\frac{\left\langle 2\left|k_{34} k_{6} k_{34}\right| 2\right]}{s_{34}-s_{234}}\right]\left(k_{3}, k_{2}\right) \\
= & \frac{1}{2}\left[\frac{\left\langle 3\left|k_{61} k_{5} k_{34}\right| 3\right]}{s_{34}-s_{345}}-\frac{\left\langle 3\left|k_{61} k_{5} k_{61}\right| 3\right]}{s_{61}-s_{234}}\right]\left(k_{6}, k_{5}\right), \\
R_{1}^{(6)}= & \frac{1}{2}\left(k_{3}, k_{5}\right)\left(k_{2}, k_{6}\right)+\frac{1}{18}\left(2\left(k_{3}, k_{6}\right)\left(k_{2}, k_{5}\right)\right. \\
& \left.-\left(k_{5}, k_{3}\right)\left(k_{2}, k_{6}\right)-\left(k_{5}, k_{6}\right)\left(k_{2}, k_{3}\right)\right) \\
+ & \frac{1}{2}\left[\frac{\left\langle 2\left|k_{45} k_{6}\left(k_{45}-k_{12}\right)\right| 2\right]}{s_{45}-s_{123}}-\frac{\left\langle 2\left|\left(k_{45}-k_{12}\right) k_{6} k_{12}\right| 2\right]}{s_{12}-s_{345}}\right]\left(k_{5}, k_{6}\right) \\
+ & \frac{1}{2}\left[\frac{\left\langle 5\left|k_{45} k_{3}\left(k_{45}-k_{12}\right)\right| 5\right]}{s_{45}-s_{345}}-\frac{\left\langle 5\left|\left(k_{45}-k_{12}\right) k_{3} k_{12}\right| 5\right]}{s_{12}-s_{123}}\right]\left(k_{2}, k_{3}\right) .(82
\end{aligned}
$$

In summary the rational part from the six-point diagram (a) in Fig. 2 is:

$$
R_{1}=\sum_{i=1}^{6} R_{1}^{(i)} .
$$

It is manifestly invariant only under a subgroup of the symmetry group, because the reduction procedure breaks the symmetry group and keeps only a subgroup manifest. Of course, $R_{1}$ must have a hidden symmetry as the final result does not depend on how one proceeds with the tensor reduction. We have checked that $R_{1}$ do have the full $Z_{6}$ symmetry.

For the diagram (b) with $i=2$ in Fig. 2, the computation proceeds much as the computation of the 6-point diagram as given in the above. The result is:

$$
\begin{aligned}
R_{2}(1) & =-I_{4}^{2 m h(4)}\left(\lambda_{6} \tilde{\lambda}_{4}, \lambda_{5} \tilde{\lambda}_{3}, \epsilon_{61}, \lambda_{3} \tilde{\lambda}_{1} ;-\left(\epsilon_{6}, \epsilon_{1}\right) / 2,-\left\langle 3\left|k_{2}\right| 1\right]\right) \\
& -\left\langle 6\left|k_{23}\right| 4\right] I_{4}^{2 m h(4)}\left(\lambda_{5} \tilde{\lambda}_{3}, \epsilon_{61}, \lambda_{3} \tilde{\lambda}_{1}\right) \\
& +\frac{[13]}{[53]}\left[\frac{4}{9}\left(\left\langle 6\left|k_{3}\right| 4\right]\left(\epsilon_{61}, k_{5}\right)+\left\langle 6\left|k_{5}\right| 4\right]\left(\epsilon_{61}, k_{3}\right)\right)\right.
\end{aligned}
$$




$$
\begin{aligned}
& -\frac{5}{9}\left(k_{3}, k_{5}\right)\left\langle 6\left|\epsilon_{61}\right| 4\right]+\frac{1}{4}\left(\left\langle 6\left|k_{3}\right| 4\right]\left(\epsilon_{61}, k_{3}\right)+\left\langle 6\left|k_{5}\right| 4\right]\left(\epsilon_{61}, k_{5}\right)\right) \\
& \left.+\frac{1}{4}\left[\frac{s_{45}+s_{345}}{s_{45}-s_{345}}\left\langle 6\left|k_{3}\right| 4\right]\left(\epsilon_{61}, k_{3}\right)+\frac{s_{34}+s_{345}}{s_{34}-s_{345}}\left\langle 6\left|k_{5}\right| 4\right]\left(\epsilon_{61}, k_{5}\right)\right]\right] \\
+ & \frac{[15]}{[35]}\left[\frac{\langle 63\rangle[34]}{2}\left(\left(\lambda_{5} \tilde{\lambda}_{3}, \epsilon_{61}\right)-\frac{\left(\lambda_{5} \tilde{\lambda}_{3}, k_{4}\right)\left(\epsilon_{61}, k_{5}\right)}{s_{45}}\right)\right. \\
+ & \left.\frac{1}{9} s_{45}\left(\tilde{\epsilon}_{45}, \epsilon_{61}\right)-\tilde{I}_{3}^{2 m(5)}\left(\lambda_{5} \tilde{\lambda}_{3}, v_{4}\right)-\tilde{I}_{3}^{2 m(5)}\left(\lambda_{5} \tilde{\lambda}_{3}, \epsilon_{61}, \lambda_{6} \tilde{\lambda}_{4}\right)\right] \\
- & \left\langle 2\left|k_{3}\right| 4\right]\left[\frac{[14]}{[54]}\left(I_{3}^{3 m}\left(\lambda_{6} \tilde{\lambda}_{2}, \epsilon_{61}\right)-\tilde{I}_{3}^{2 m(4)}\left(\lambda_{6} \tilde{\lambda}_{2}, \epsilon_{61}\right)\right)\right. \\
+ & \left.\frac{[15]}{[45]} I_{4}^{2 m h(4)}\left(\lambda_{5} \tilde{\lambda}_{4}, \lambda_{6} \tilde{\lambda}_{2}, \epsilon_{61}\right)-I_{4}^{2 m e(2)}\left(\lambda_{6} \tilde{\lambda}_{2}, \lambda_{5} \tilde{\lambda}_{1}, \epsilon_{61}\right)\right]
\end{aligned}
$$

where

$$
\begin{aligned}
v_{4} & =\left(\lambda_{6} \tilde{\lambda}_{4}, k_{3}\right) \epsilon_{61}-\frac{1}{2}\left(\epsilon_{6}, \epsilon_{1}\right) \lambda_{6} \tilde{\lambda}_{4}, \\
\tilde{\epsilon}_{45} & =\left.\epsilon_{45}\right|_{\epsilon_{4} \rightarrow \lambda_{6}} \tilde{\lambda}_{4}= \\
& =\frac{\langle 65\rangle}{\langle 45\rangle} \lambda_{5} \tilde{\lambda}_{3}-\frac{[34]}{[54]} \lambda_{6} \tilde{\lambda}_{4}+\frac{\langle 65\rangle[34]}{2\langle 45\rangle[54]}\left(k_{4}-k_{5}\right) .
\end{aligned}
$$

There are three different kinds of box diagrams shown in Fig. 2. First, the rational part of the 2-mass-hard box with two massless external lines $k_{2,3}$ is:

$$
R_{3}(1)=I_{4}^{2 m h(2)}\left(\epsilon_{2}, \epsilon_{3}, \epsilon_{45}, \epsilon_{61} ;-\left(\epsilon_{4}, \epsilon_{5}\right) / 2,-\left(\epsilon_{6}, \epsilon_{1}\right) / 2\right) .
$$

The explicit expression in terms of the rational parts of triangle integrals and other more primitive quantities is given in the appendix.

The rational part of the two-mass-easy box with massless external lines $k_{2,5}$ is decomposed into two parts $R_{4}(1)+R_{4}(4)$ and we have:

$$
\begin{aligned}
R_{4}(1) & =\frac{\langle 62\rangle[35]}{\langle 52\rangle[25]}\left[\frac{\left\langle 5\left|k_{34}\right| 2\right]^{2}\left(\epsilon_{34}, k_{2}\right)\left(\epsilon_{61}, k_{2}\right)}{3\left(s_{34}-s_{234}\right)\left(s_{61}-s_{345}\right)}+\frac{5}{18}\left\langle 5\left|\epsilon_{34}\right| 2\right]\left\langle 5\left|\epsilon_{61}\right| 2\right]\right] \\
& +\frac{[32]}{[52]}\left[I_{4}^{2 m e(2)}\left(\epsilon_{2}, \epsilon_{34}, k_{5}, \epsilon_{61}\right)\right. \\
& \left.+\frac{1}{4}\left[\frac{\left(\epsilon_{2}, k_{34}\right)}{s_{34}-s_{234}}-\frac{\left(\epsilon_{2}, k_{61}\right)}{s_{61}-s_{345}}\right]\left(\left(\epsilon_{3}, \epsilon_{4}\right) \epsilon_{61}+\left(\epsilon_{6}, \epsilon_{1}\right) \epsilon_{34}, k_{2}\right)\right]
\end{aligned}
$$




$$
\begin{aligned}
- & \frac{\langle 65\rangle[32]}{36\langle 25\rangle[52]}\left(2\left(k_{2}, k_{5}\right)\left(\epsilon_{34}, \epsilon_{61}\right)\right. \\
& \left.-\left(\left(\epsilon_{34}, k_{2}\right)\left(\epsilon_{61}, k_{5}\right)+\left(\epsilon_{34}, k_{5}\right)\left(\epsilon_{61}, k_{2}\right)\right)\right) .
\end{aligned}
$$

$R_{4}(4)$ is obtained from $R_{4}(1)$ by the symmetry operation $\tau^{3}$. The rational part from one of the one-mass box diagrams, i.e. diagram (e) with $i=2$ in Fig. 2, is:

$$
\begin{aligned}
R_{5}(1)= & \frac{\langle 62\rangle}{\langle 42\rangle}\left[\frac{4}{9}\left(\left(\epsilon_{3}, k_{2}\right)\left(\epsilon_{561}, k_{4}\right)+\left(\epsilon_{3}, k_{4}\right)\left(\epsilon_{561}, k_{2}\right)\right)\right. \\
& -\frac{5}{9}\left(k_{2}, k_{4}\right)\left(\epsilon_{3}, \epsilon_{561}\right)+\frac{1}{4}\left(\left(\epsilon_{3}, k_{2}\right)\left(\epsilon_{561}, k_{2}\right)+\left(\epsilon_{3}, k_{4}\right)\left(\epsilon_{561}, k_{4}\right)\right) \\
+ & \left.\frac{1}{4}\left[\frac{s_{34}+s_{234}}{s_{34}-s_{234}}\left(\epsilon_{3}, k_{2}\right)\left(\epsilon_{561}, k_{2}\right)+\frac{s_{23}+s_{234}}{s_{23}-s_{234}}\left(\epsilon_{3}, k_{4}\right)\left(\epsilon_{561}, k_{4}\right)\right]\right] \\
+ & \frac{\langle 64\rangle}{\langle 24\rangle}\left[\frac{1}{2}\left(\left(\epsilon_{5}, \epsilon_{61}\right)+\left(\epsilon_{56}, \epsilon_{1}\right)\right) \tilde{I}_{3}^{2 m(4)}\left(\epsilon_{4}, \epsilon_{3}\right)\right. \\
& \left.\quad+\frac{1}{9} s_{34}\left(\epsilon_{34}, \epsilon_{561}\right)-\tilde{I}_{3}^{2 m(4)}\left(\epsilon_{4}, \epsilon_{561}, \epsilon_{3}\right)\right] .
\end{aligned}
$$

The rational part for the three-mass triangle with external momenta $\left(k_{23}, k_{45}, k_{61}\right)$, i.e. diagram $(\mathrm{g})$ in Fig. 2 , is:

$$
\begin{aligned}
R_{0} & =I_{3}^{3 m}\left(\epsilon_{23}, \epsilon_{45}, \epsilon_{61}\right)-\frac{1}{2}\left(\epsilon_{2}, \epsilon_{3}\right) I_{3}^{3 m}\left(\epsilon_{45}, \epsilon_{61}\right) \\
& -\frac{1}{2}\left(\epsilon_{4}, \epsilon_{5}\right) I_{3}^{3 m}\left(\epsilon_{61}, \epsilon_{23}\right)-\frac{1}{2}\left(\epsilon_{6}, \epsilon_{1}\right) I_{3}^{3 m}\left(\epsilon_{23}, \epsilon_{45}\right) .
\end{aligned}
$$

The rational part from the 2 two-mass triangle diagrams, i.e. diagrams (h) and (i) with $i=2$ in Fig. 2, is:

$$
\begin{aligned}
R_{6}(1) & =I_{3}^{2 m(2)}\left(\epsilon_{2}, \epsilon_{34}, \epsilon_{561}\right)-\frac{1}{2} I_{3}^{2 m(2)}\left(\epsilon_{2}, v_{6}\right) \\
& +\tilde{I}_{3}^{2 m(2)}\left(\epsilon_{2}, \epsilon_{345}, \epsilon_{61}\right)-\frac{1}{2} \tilde{I}_{3}^{2 m(2)}\left(\epsilon_{2}, \tilde{v}_{6}\right) \\
v_{6} & =\left(\epsilon_{3}, \epsilon_{4}\right) \epsilon_{561}+\left(\left(\epsilon_{5}, \epsilon_{61}\right)+\left(\epsilon_{56}, \epsilon_{1}\right)\right) \epsilon_{34}, \\
\tilde{v}_{6} & =\left(\left(\epsilon_{3}, \epsilon_{45}\right)+\left(\epsilon_{34}, \epsilon_{5}\right)\right) \epsilon_{61}+\left(\epsilon_{6}, \epsilon_{1}\right) \epsilon_{345} .
\end{aligned}
$$

All the one-mass triangle diagrams combined with the corresponding bubble diagrams give a vanishing contribution to the rational part, as all two 
neighboring gluons have opposite helicities for this helicity configuration. The

remaining 3 bubble diagrams give the following contribution to the rational part:

$$
R_{8}=-\frac{1}{9}\left(s_{123}\left(\epsilon_{123}, \epsilon_{456}\right)+s_{234}\left(\epsilon_{234}, \epsilon_{561}\right)+s_{345}\left(\epsilon_{345}, \epsilon_{612}\right)\right) .
$$

Adding all the above results with appropriate symmetry operations, the final result for the rational part is:

$$
R=F\left[R_{0}+\left(\left.R_{0}\right|_{i \rightarrow i+1}\right)^{*}+R_{1}+R_{8}+\sum_{i=2}^{6} \sum_{j=1}^{6} R_{i}(j)\right],
$$

where $R_{i}(j)=\tau^{j-1}\left(R_{i}(1)\right)$ and

$$
F=\frac{1}{\langle 62\rangle\langle 24\rangle\langle 46\rangle[15][31][53]}
$$

is an overall factor.

\section{Factorization and the NMHV amplitudes}

The results obtained in previous 2 sections for the rational parts of the 2 NMHV amplitudes are quite complicated analytic expressions. To present the results in a readable form we have used 3 levels of definitions. The first level consists of all the scalar products (including all kinematic variables) in terms of spinor products. The second level of definition consists of the composite polarization vectors $\epsilon_{i(i+1) \ldots .}$. The last level consists of the rational parts of box and triangle integrals in terms of some elementary functions.

If we make a naive expansion of all the level 2 and 3 definitions, the resulting analytic expression is actually quite large. Although our results were derived rigorously from first principle, it would be best if we can make some independent checks for these results, especially to detect some human errors or typos. A trivial but quite useful check is that the rational part should have the correct spinor weight [18].

One stringent check is factorization. The factorization in one-loop gauge theory was discussed in detail by Bern and Chalmers in [47]. The 2-particle 
factorization properties were discussed in $[4,36]$. As two momenta become collinear the one-loop amplitudes behave as $[4,15]$

$$
\begin{aligned}
A_{n}^{1-\text { loop } \longrightarrow} & \sum_{\lambda= \pm}\left(\operatorname{Split}_{-\lambda}^{\text {tree }}\left(a^{\lambda_{a}}, b^{\lambda_{b}}\right) A_{n-1}^{1-\text { loop }}\left(\cdots(a+b)^{\lambda} \cdots\right)\right. \\
& \left.+\operatorname{Split}_{-\lambda}^{1-\text { loop }}\left(a^{\lambda_{a}}, b^{\lambda_{b}}\right) A_{n-1}^{\text {tree }}\left(\cdots(a+b)^{\lambda} \cdots\right)\right),
\end{aligned}
$$

where $k_{a} \rightarrow z K$ and $k_{b} \rightarrow(1-z) K$, with $K=k_{a}+k_{b}, K^{2}=s_{a b} \rightarrow 0$. A similar relation also holds for multi-particle factorization. The tree and 1loop splitting amplitudes in massless QCD have been given in $[15,47,20]$. As we have a complete knowledge of the 4- and 5-gluon amplitudes at tree and one-loop level, it is straightforward to derive a set of relations which must be satisfied by the 6 -gluon amplitude. These relations provide an extremely stringent check.

Berger, Bern, Dixon, Forde and Kosower have done this check by using their factorization check program and found that the results given in the previous two sections in fact pass the check for all channels. The Mathematica codes used by them were input by us by using the formulas given in this paper. The codes are available up to requests and/or they will be stored somewhere for free use.

\section{Conclusion}

In this paper, by using the formalism developed in a previous paper [1], we computed explicitly the rational parts of the 6-gluon one-loop amplitudes for all the possible helicity configurations. In particular we present most of the intermediate results for the four helicity configurations which have no available published results in the literature. For two of the MHV helicity configurations our results agree with the all multiplicity results [9] which are obtained by using the bootstrap recursive approach of Bern, Dixon and Kosower [6, 7]. For other two non-MHV helicity configurations our results passed the factorization tests in all channels. By combining our results with the previously computed cut-constructible parts [33], we have all the ingredients for a complete expression of the 6-gloun one-loop QCD amplitude. This paves the way for assembling new NLO helicity amplitudes in numerical codes for cross-section calculations. 
The method presented in this paper can be applied straightforwardly to compute all the rational parts of the 6-parton one-loop QCD amplitudes. Apart from the rational parts of the 3-mass tensor box integrals, our method can also be applied to compute the rational parts for $e^{+} e^{-} \rightarrow 5$-partons. As we demonstrated in this paper all the intermediate steps of our computations are carried out by hand. Only for the input of composite polarization vectors and for combining all the results together (by using various permutation symmetry operations) we used computer symbolic system Mathematica. There should be no much difficulty to automate our method (to compute the rational parts). In this way we may push the limit to 8-gluon or higher.

\section{Appendix: Formulas for the rational parts of some Feynman integrals}

For quick reference we list here the explicit formulas for the rational parts of some Feynman integrals. The derivation can be found in [1].

Firstly, for the bubble integral we have:

$$
\begin{aligned}
I_{2}\left(\epsilon_{1}, \epsilon_{2}\right) & =\int \frac{\mathrm{d}^{D} p}{i \pi^{D / 2}} \frac{\left(\epsilon_{1}, p\right)\left(\epsilon_{2}, p\right)}{p^{2}(p+K)^{2}} \\
& =\frac{1}{18}\left(\left(\epsilon_{1}, K\right)\left(\epsilon_{2}, K\right)-2 K^{2}\left(\epsilon_{1}, \epsilon_{2}\right)\right) .
\end{aligned}
$$

where $K$ is the sum of momenta on one side of the bubble diagram.

For three-mass triangle integral with external momenta $\left\{K_{1}, K_{2}, K_{3}\right\}$ and arbitrary polarization vectors $\epsilon_{1,2,3}$ we have

$$
\begin{aligned}
I_{3}\left(\epsilon_{1}, \epsilon_{2}, \epsilon_{3}\right) \equiv & \int \frac{\mathrm{d}^{D} p}{i \pi^{D / 2}} \frac{\left(\epsilon_{1}, p\right)\left(\epsilon_{2}, p-K_{1}\right)\left(\epsilon_{3}, p+K_{3}\right)}{p^{2}\left(p-K_{1}\right)^{2}\left(p+K_{3}\right)^{2}} \\
= & -F_{0}\left(s_{1}, s_{2}, s_{3}\right)\left(\left(\epsilon_{1}, K_{1}\right)\left(\epsilon_{2}, K_{1}\right)\left(\epsilon_{3}, K_{2}\right)\right. \\
& +\left(\epsilon_{1}, K_{3}\right)\left(\epsilon_{2}, K_{2}\right)\left(\epsilon_{3}, K_{2}\right)+\left(\epsilon_{1}, K_{3}\right)\left(\epsilon_{2}, K_{1}\right)\left(\epsilon_{3}, K_{3}\right) \\
& \left.+\left(\epsilon_{1}, K_{3}\right)\left(\epsilon_{2}, K_{1}\right)\left(\epsilon_{3}, K_{2}\right)-\left(\epsilon_{1}, K_{1}\right)\left(\epsilon_{2}, K_{2}\right)\left(\epsilon_{3}, K_{3}\right)\right) \\
- & \sum_{i=1}^{3}\left(\epsilon_{1}, K_{i}\right)\left(\epsilon_{2}, K_{i}\right)\left(\epsilon_{3}, K_{i}\right) F_{i}\left(s_{1}, s_{2}, s_{3}\right) \\
- & \frac{1}{2 \Delta}\left(\left(s_{1}-s_{2}-s_{3}\right)\left(\epsilon_{1}, K_{1}\right)\left(\epsilon_{2}, K_{1}\right)\left(\epsilon_{3}, K_{3}\right)\right.
\end{aligned}
$$




$$
\begin{aligned}
& +\left(s_{2}-s_{3}-s_{1}\right)\left(\epsilon_{1}, K_{1}\right)\left(\epsilon_{2}, K_{2}\right)\left(\epsilon_{3}, K_{2}\right) \\
& \left.+\left(s_{3}-s_{1}-s_{2}\right)\left(\epsilon_{1}, K_{3}\right)\left(\epsilon_{2}, K_{2}\right)\left(\epsilon_{3}, K_{3}\right)\right) \\
& +\frac{7}{36}\left(\left(\epsilon_{1}, \epsilon_{2}\right)\left(\epsilon_{3}, K_{3}-K_{2}\right)+\left(\epsilon_{2}, \epsilon_{3}\right)\left(\epsilon_{1}, K_{1}-K_{3}\right)\right. \\
& \left.\quad+\left(\epsilon_{3}, \epsilon_{1}\right)\left(\epsilon_{2}, K_{2}-K_{1}\right)\right) \\
& +\frac{1}{12 \Delta}\left(\left(\epsilon_{1}, \epsilon_{2}\right)\left(\epsilon_{3}, K_{3}-K_{2}\right) s_{1}\left(s_{2}+s_{3}-s_{1}\right)\right. \\
& \quad+\left(\epsilon_{2}, \epsilon_{3}\right)\left(\epsilon_{1}, K_{1}-K_{3}\right) s_{2}\left(s_{3}+s_{1}-s_{2}\right) \\
& \left.+\left(\epsilon_{3}, \epsilon_{1}\right)\left(\epsilon_{2}, K_{2}-K_{1}\right) s_{3}\left(s_{1}+s_{2}-s_{3}\right)\right) \\
& +\frac{1}{12 \Delta}\left(\left(\epsilon_{1}, \epsilon_{2}\right)\left(\epsilon_{3}, K_{1}\right)\left(s_{3}-s_{2}\right)\left(s_{2}+s_{3}-s_{1}\right)\right. \\
& \quad+\left(\epsilon_{2}, \epsilon_{3}\right)\left(\epsilon_{1}, K_{2}\right)\left(s_{1}-s_{3}\right)\left(s_{3}+s_{1}-s_{2}\right) \\
& \left.+\left(\epsilon_{3}, \epsilon_{1}\right)\left(\epsilon_{2}, K_{3}\right)\left(s_{2}-s_{1}\right)\left(s_{1}+s_{2}-s_{3}\right)\right),
\end{aligned}
$$

where

$$
\begin{aligned}
F_{0}\left(s_{1}, s_{2}, s_{3}\right) & =\frac{10 s_{1} s_{2} s_{3}}{3 \Delta^{2}}+\frac{\left(s_{1}+s_{2}+s_{3}\right)}{6 \Delta} \\
F_{1}\left(s_{1}, s_{2}, s_{3}\right) & =\frac{5\left(s_{1}+s_{2}-s_{3}\right) s_{2} s_{3}}{3 \Delta^{2}}+\frac{\left(s_{1}-s_{3}\right)}{3 \Delta} \\
F_{2}\left(s_{1}, s_{2}, s_{3}\right) & =\frac{5\left(s_{2}+s_{3}-s_{1}\right) s_{3} s_{1}}{3 \Delta^{2}}+\frac{\left(s_{2}-s_{1}\right)}{3 \Delta} \\
F_{3}\left(s_{1}, s_{2}, s_{3}\right) & =\frac{5\left(s_{3}+s_{1}-s_{2}\right) s_{1} s_{2}}{3 \Delta^{2}}+\frac{\left(s_{3}-s_{2}\right)}{3 \Delta} \\
\Delta & =s_{1}^{2}+s_{2}^{2}+s_{3}^{2}-2\left(s_{1} s_{2}+s_{2} s_{3}+s_{3} s_{1}\right)
\end{aligned}
$$

and $s_{i}=K_{i}^{2}$.

For degree 2 polynomial we have:

$$
\begin{aligned}
I_{3}\left(\epsilon_{i}, \epsilon_{j}\right) & \equiv \int \frac{\mathrm{d}^{D} p}{i \pi^{D / 2}} \frac{\left(\epsilon_{i}, p\right)\left(\epsilon_{j}, p\right)}{p^{2}\left(p-K_{1}\right)^{2}\left(p+K_{3}\right)^{2}} \\
& =-\frac{1}{2 \Delta}\left(s_{1}\left(\left(\epsilon_{i}, K_{2}\right)\left(\epsilon_{j}, K_{3}\right)+\left(\epsilon_{i}, K_{3}\right)\left(\epsilon_{j}, K_{2}\right)\right)\right. \\
& +s_{2}\left(\left(\epsilon_{i}, K_{3}\right)\left(\epsilon_{j}, K_{1}\right)+\left(\epsilon_{i}, K_{1}\right)\left(\epsilon_{j}, K_{3}\right)\right) \\
& \left.+s_{3}\left(\left(\epsilon_{i}, K_{1}\right)\left(\epsilon_{j}, K_{2}\right)+\left(\epsilon_{i}, K_{2}\right)\left(\epsilon_{j}, K_{1}\right)\right)\right)+\frac{1}{2}\left(\epsilon_{i}, \epsilon_{j}\right) .
\end{aligned}
$$


For 2-mass triangle integrals the above formulas simplify greatly. They are:

$$
\begin{aligned}
I_{3}\left(\epsilon_{1}, \epsilon_{2}\right) & \equiv \int \frac{\mathrm{d}^{D} p}{i \pi^{D / 2}} \frac{\left(\epsilon_{1}, p\right)\left(\epsilon_{2}, p\right)}{p^{2}\left(p-k_{1}\right)^{2}\left(p+K_{3}\right)^{2}} \\
& =\frac{1}{2}\left(\epsilon_{1}, \epsilon_{2}\right)+\frac{\left(K_{2}^{2}+K_{3}^{2}\right)}{2\left(K_{2}^{2}-K_{3}^{2}\right)^{2}}\left(\epsilon_{1}, k_{1}\right)\left(\epsilon_{2}, k_{1}\right) \\
& +\frac{\left(\left(\epsilon_{1}, K_{2}\right)\left(\epsilon_{2}, k_{1}\right)-\left(\epsilon_{1}, k_{1}\right)\left(\epsilon_{2}, K_{3}\right)\right)}{2\left(K_{2}^{2}-K_{3}^{2}\right)}, \\
I_{3}\left(\epsilon_{1}, \epsilon_{2}\right) & =\frac{1}{2}\left(\epsilon_{1}, \epsilon_{2}\right)+\frac{\left(\epsilon_{1}, K_{2}\right)\left(\epsilon_{2}, k_{1}\right)}{2\left(K_{2}^{2}-K_{3}^{2}\right)}, \quad\left(\epsilon_{1}, k_{1}\right)=0,
\end{aligned}
$$

and

$$
\begin{aligned}
I_{3}\left(\epsilon_{i}\right) & \equiv \int \frac{\mathrm{d}^{D} p}{i \pi^{D / 2}} \frac{\left(\epsilon_{1}, p\right)\left(\epsilon_{2}, p-k_{1}\right)\left(\epsilon_{3}, p\right)}{p^{2}\left(p-k_{1}\right)^{2}\left(p+K_{3}\right)^{2}} \\
& =\frac{1}{36}\left(\left(\epsilon_{2}, 4 K_{2}-7 k_{1}\right)\left(\epsilon_{1}, \epsilon_{3}\right)-(2 \leftrightarrow 3)+4\left(\epsilon_{1}, K_{2}\right)\left(\epsilon_{2}, \epsilon_{3}\right)\right) \\
& -\frac{\left(K_{2}^{2}+K_{3}^{2}\right)}{6\left(K_{2}^{2}-K_{3}^{2}\right)^{2}}\left(\epsilon_{1}, K_{2}\right)\left(\epsilon_{2}, k_{1}\right)\left(\epsilon_{3}, k_{1}\right) \\
& -\frac{\left(\epsilon_{1}, K_{2}\right)\left(\left(\epsilon_{2}, k_{1}\right)\left(\epsilon_{3}, K_{3}\right)-\left(\epsilon_{2}, K_{2}\right)\left(\epsilon_{3}, k_{1}\right)\right)}{6\left(K_{2}^{2}-K_{3}^{2}\right)} \\
& -\frac{\left(K_{2}^{2}+K_{3}^{2}\right)}{12\left(K_{2}^{2}-K_{3}^{2}\right)}\left(\left(\epsilon_{1}, \epsilon_{2}\right)\left(\epsilon_{3}, k_{1}\right)+\left(\epsilon_{1}, \epsilon_{3}\right)\left(\epsilon_{2}, k_{1}\right)\right)
\end{aligned}
$$

where $\epsilon_{1}$ satisfies the physical condition $\left(\epsilon_{1}, k_{1}\right)=0$ and $\epsilon_{2,3}$ are arbitrary 4-dimensional polarization vectors.

For degree 3 two-mass-easy box integrals we have:

$$
I_{4}^{2 m e}\left(\lambda_{3} \tilde{\lambda}_{1}, \epsilon_{2}, \epsilon_{3}\right)=\frac{\left\langle 3\left|K_{2}\right| 1\right]}{2}\left[\frac{\left(\epsilon_{2}, k_{3}\right)\left(\epsilon_{3}, k_{3}\right)}{\left(K_{2}^{2}-t\right)\left(K_{4}^{2}-s\right)}-\left(k_{3} \rightarrow k_{1}, s \leftrightarrow t\right)\right] .
$$

If two of the polarization vectors satisfy the physical condition, i.e. $\left(\epsilon_{1}, k_{1}\right)=$ 0 and $\left(\epsilon_{3}, k_{3}\right)=0$, we have

$$
\begin{aligned}
I_{4}^{2 m e}\left(\epsilon_{1}, \epsilon_{2}, \epsilon_{3}\right) & =-\frac{\left(\epsilon_{1}, k_{3}\right)\left(\epsilon_{3}, k_{1}\right)}{2\left(k_{1}, k_{3}\right)}\left[\frac{\left(\epsilon_{2}, k_{3}\right)}{K_{2}^{2}-t}+\frac{\left(\epsilon_{2}, k_{1}\right)}{K_{4}^{2}-t}\right] \\
& -\frac{\left(\epsilon_{1}, K_{2}\right)\left(\epsilon_{2}, k_{1}\right)\left(\epsilon_{3}, k_{1}\right)}{2\left(K_{2}^{2}-s\right)\left(K_{4}^{2}-t\right)}-\frac{\left(\epsilon_{1}, k_{3}\right)\left(\epsilon_{2}, k_{3}\right)\left(\epsilon_{3}, K_{4}\right)}{2\left(K_{2}^{2}-t\right)\left(K_{4}^{2}-s\right)} .
\end{aligned}
$$


The formulas for the degree 3 two-mass-hard box integrals are:

$$
\begin{aligned}
& I_{4}^{2 m h}\left(\lambda_{1} \tilde{\lambda}_{2}, \epsilon_{2}, \epsilon_{3}\right)=\frac{\left\langle 1\left|K_{3}\right| 2\right]}{4 \delta} I_{4}\left(\epsilon_{2}, \epsilon_{3}\right), \\
& I_{4}^{2 m h}\left(\lambda_{2} \tilde{\lambda}_{1}, \epsilon_{2}, \epsilon_{3}\right)=\frac{\left\langle 2\left|K_{3}\right| 1\right]}{4 \delta} I_{4}\left(\epsilon_{2}, \epsilon_{3}\right),
\end{aligned}
$$

where

$$
\begin{aligned}
I_{4}\left(\epsilon_{2}, \epsilon_{3}\right)= & \left(\epsilon_{2}, k_{1}\right)\left(\epsilon_{3}, K_{4}\right)+\left(\epsilon_{2}, K_{4}\right)\left(\epsilon_{3}, k_{1}\right)+\left(\epsilon_{2}, k_{2}\right)\left(\epsilon_{3}, K_{3}\right) \\
+ & \left(\epsilon_{2}, K_{3}\right)\left(\epsilon_{3}, k_{2}\right)-\frac{1}{\Delta}\left[2\left(K_{3}^{2} K_{4}^{2}-t^{2}+\delta\right)\left(\epsilon_{2}, k_{12}\right)\left(\epsilon_{3}, k_{12}\right)\right. \\
+ & \left(K_{3}^{2}+K_{4}^{2}-s-2 t\right)\left(\left(K_{3}^{2}-K_{4}^{2}+s\right)\left(\epsilon_{2}, K_{4}\right)\left(\epsilon_{3}, K_{4}\right)\right. \\
& \left.\left.+\left(K_{4}^{2}-K_{3}^{2}+s\right)\left(\epsilon_{2}, K_{3}\right)\left(\epsilon_{3}, K_{3}\right)\right)\right] \\
+ & \frac{K_{4}^{2}+t}{K_{4}^{2}-t}\left(\epsilon_{2}, k_{1}\right)\left(\epsilon_{3}, k_{1}\right)+\frac{K_{3}^{2}+t}{K_{3}^{2}-t}\left(\epsilon_{2}, k_{2}\right)\left(\epsilon_{3}, k_{2}\right)
\end{aligned}
$$

and

$$
\begin{aligned}
\delta & =K_{3}^{2} K_{4}^{2}-\left(K_{3}^{2}+K_{4}^{2}\right) t+(s+t) t \\
\Delta & =\Delta\left(k_{12}^{2}, K_{3}^{2}, K_{4}^{2}\right) \\
\Delta\left(s_{1}, s_{2}, s_{3}\right) & =s_{1}^{2}+s_{2}^{2}+s_{3}^{2}-2\left(s_{1} s_{2}+s_{2} s_{3}+s_{3} s_{1}\right),
\end{aligned}
$$

are functions of the external momentum invariants.

In order to give the formulas for the rational parts of the degree 4 polynomials, we define:

$$
I_{4}\left(\epsilon_{1}, \epsilon_{2}, \epsilon_{3}, \epsilon_{4}\right) \equiv \int \frac{\mathrm{d}^{D} p}{i \pi^{D / 2}} \frac{\left(\epsilon_{1}, p\right)\left(\epsilon_{2}, p-K_{1}\right)\left(\epsilon_{3}, p-K_{12}\right)\left(\epsilon_{4}, p+K_{4}\right)}{p^{2}\left(p-K_{1}\right)^{2}\left(p-K_{12}\right)^{2}\left(p+K_{4}\right)^{2}},
$$

where $K_{12}=K_{1}+K_{2}$. For two-mass-easy cases $\left(K_{i, 3}=k_{1,3}\right.$ are massless external lines), we have

$$
\begin{aligned}
I_{4}\left(\lambda_{3} \tilde{\lambda}_{1}, \epsilon_{2}, \lambda_{1} \tilde{\lambda}_{3}, \epsilon_{4}\right) & =-\frac{1}{4}\left(\frac{K_{2}^{2}+s}{K_{2}^{2}-s}+\frac{K_{4}^{2}+t}{K_{4}^{2}-t}\right)\left(\epsilon_{2}, k_{1}\right)\left(\epsilon_{4}, k_{1}\right) \\
- & \frac{1}{4}\left(\frac{K_{2}^{2}+t}{K_{2}^{2}-t}+\frac{K_{4}^{2}+s}{K_{4}^{2}-s}\right)\left(\epsilon_{2}, k_{3}\right)\left(\epsilon_{4}, k_{3}\right)-\frac{5}{9}\left(k_{1}, k_{3}\right)\left(\epsilon_{2}, \epsilon_{4}\right)
\end{aligned}
$$




$$
\begin{aligned}
&+ \frac{4}{9}\left(\left(\epsilon_{2}, k_{1}\right)\left(\epsilon_{4}, k_{3}\right)+\left(\epsilon_{2}, k_{3}\right)\left(\epsilon_{4}, k_{1}\right)\right) \\
& I_{4}\left(\lambda_{1} \tilde{\lambda}_{3}, \epsilon_{2}, \lambda_{1} \tilde{\lambda}_{3}, \epsilon_{4}\right)= \frac{5}{9}\left\langle 1\left|\epsilon_{2}\right| 3\right]\left\langle 1\left|\epsilon_{4}\right| 3\right] \\
&+\frac{\left\langle 1\left|K_{2}\right| 3\right]^{2}}{3}\left[\frac{\left(\epsilon_{2}, k_{1}\right)\left(\epsilon_{4}, k_{1}\right)}{\left(K_{2}^{2}-s\right)\left(K_{4}^{2}-t\right)}+\frac{\left(\epsilon_{2}, k_{3}\right)\left(\epsilon_{4}, k_{3}\right)}{\left(K_{2}^{2}-t\right)\left(K_{4}^{2}-s\right)}\right] .
\end{aligned}
$$

Other cases can be either obtained by conjugation or relabelling $k_{1,3}$.

For 2-mass-hard box cases $\left(K_{1,2}=k_{1,2}\right.$ are two massless external lines), we define:

$$
\begin{aligned}
I_{4}^{2 m h} & \left(\epsilon_{1}, \epsilon_{2}, \epsilon_{3}, \epsilon_{4} ; c_{3}, c_{4}\right) \\
& \equiv I_{4}\left[\left(\epsilon_{1}, p\right)\left(\epsilon_{2}, p-k_{1}\right)\left(\left(\epsilon_{3}, p+K_{4}\right)+c_{3}\right)\left(\left(\epsilon_{4}, p+K_{4}\right)+c_{4}\right)\right] \\
& =\int \frac{\mathrm{d}^{D} p}{i \pi^{D / 2}} \frac{\left(\epsilon_{1}, p\right)\left(\epsilon_{2}, p-k_{1}\right)\left(\left(\epsilon_{3}, p+K_{4}\right)+c_{3}\right)\left(\left(\epsilon_{4}, p+K_{4}\right)+c_{4}\right)}{p^{2}\left(p-k_{1}\right)^{2}\left(p-k_{12}\right)^{2}\left(p+K_{4}\right)^{2}},
\end{aligned}
$$

and

$$
\begin{aligned}
I_{4}^{2 m h}\left(\lambda_{1} \tilde{\eta}_{1}, \eta_{2} \tilde{\lambda}_{2}, \epsilon_{3}, \epsilon_{4} ; c_{3}, c_{4}\right) \\
=\frac{1}{\left\langle 2\left|K_{3}\right| 1\right]}\left[-\frac{1}{6}\left\langle\eta_{2}\left|k_{2} K_{3} k_{1}\right| \tilde{\eta}_{1}\right\rangle\left(\epsilon_{3}, \epsilon_{4}\right)-t\left\langle\eta_{2} 2\right\rangle\left[\tilde{\eta}_{1} 1\right] I_{4}^{2 m h}\left(\lambda_{1} \tilde{\lambda}_{2}, \epsilon_{3}, \epsilon_{4}\right)\right. \\
+\quad t\left\langle\eta_{2}\left|k_{2}\right| \tilde{\eta}_{1}\right] I_{3}^{3 m}\left(\epsilon_{3}, \epsilon_{4}\right)+\left\langle\eta_{2} 2\right\rangle\left[\tilde{\eta}_{1} 1\right]\left(\frac{1}{2}\left(\left\langle 1\left|\epsilon_{3}\right| 2\right] c_{4}+\left\langle 1\left|\epsilon_{4}\right| 2\right] c_{3}\right)\right. \\
\left.\quad+\frac{1}{18}\left(\left\langle 1\left|\epsilon_{3}\right| 2\right] \epsilon_{4}+\left\langle 1\left|\epsilon_{4}\right| 2\right] \epsilon_{3}, 7 k_{1}+2 k_{2}+9 K_{4}\right)\right) \\
\quad+\frac{1}{18}\left\langle\eta_{2} 2\right\rangle\left[\tilde{\eta}_{1} 2\right]\left(\left(\epsilon_{3}, k_{12}\right)\left(\epsilon_{4}, k_{12}\right)-2 s_{12}\left(\epsilon_{3}, \epsilon_{4}\right)\right) \\
\quad+\frac{1}{18}\left\langle\eta_{2}\left|\left(k_{2}+K_{3}\right)\right| \tilde{\eta}_{1}\right]\left(\left(\epsilon_{3}, k_{2}+K_{3}\right)\left(\epsilon_{4}, k_{2}+K_{3}\right)-2 t\left(\epsilon_{3}, \epsilon_{4}\right)\right) \\
\quad-\frac{1}{18}\left\langle\eta_{2}\left|K_{3}\right| \tilde{\eta}_{1}\right]\left(\left(\epsilon_{3}, K_{3}\right)\left(\epsilon_{4}, K_{3}\right)-2 K_{3}^{2}\left(\epsilon_{3}, \epsilon_{4}\right)\right) \\
+\quad\left\langle 2\left|K_{3}\right| \tilde{\eta}_{1}\right\rangle\left(I_{3}^{2 m}\left(\eta_{2} \tilde{\lambda}_{2}, \epsilon_{3}, \epsilon_{4}\right)\right. \\
\left.\quad+\quad I_{3}^{2 m}\left(\eta_{2} \tilde{\lambda}_{2},\left(c_{3}-\left(\epsilon_{3}, K_{3}\right)\right) \epsilon_{4}+\left(c_{4}+\left(\epsilon_{4}, K_{4}+k_{1}\right)\right) \epsilon_{3}\right)\right) \\
\quad+\quad I_{3}^{3 m}\left(v, \epsilon_{3}, \epsilon_{4}\right)+I_{3}^{3 m}\left(v,\left(c_{3}-\left(\epsilon_{3}, K_{3}\right)\right) \epsilon_{4}+c_{4} \epsilon_{3}\right) \\
\quad+\left\langle\eta_{2}\left|K_{4}\right| 1\right]\left(\tilde{I}_{3}^{2 m}\left(\lambda_{1} \tilde{\eta}_{1}, \epsilon_{3}, \epsilon_{4}\right)\right. \\
\left.+\quad \tilde{I}_{3}^{2 m}\left(\lambda_{1} \tilde{\eta}_{1},\left(c_{3}-\left(\epsilon_{3}, k_{2}+K_{3}\right)\right) \epsilon_{4}+\left(c_{4}+\left(\epsilon_{4}, K_{4}\right)\right) \epsilon_{3}\right)\right]
\end{aligned}
$$


where

$$
v=\left\langle\eta_{2}\left|K_{3}\right| 1\right] \lambda_{1} \tilde{\eta}_{1}+\left\langle\eta_{2}\left|K_{3}\right| 2\right] \lambda_{2} \tilde{\eta}_{1}-\left(k_{2}, K_{3}\right) \eta_{2} \tilde{\eta}_{1} .
$$

The opposite helicity case is obtained by conjugation.

\section{Acknowledgments}

We would like to thank Carola F. Berger, Zvi Bern, Lance J. Dixon, Darren Forde and David A. Kosower for sending us their results prior to publication [9], and for discussions, reading the paper, comments and assistance in comparing our six-gluon results with theirs. We also thank them for checking the NMHV results by using their factorization check program. CJZ would like to thank J. -P. Ma for constant encouragements, helpful discussions and careful reading of the paper. His (financial) support (to buy a computer which was still in use today) actually goes back to the much earlier difficult times when I did not have enough grants from other sources. What is more important is that there are no strings attached to his support and it is up to the last author to explore what he wants to. CJZ would also like to thank R. Iengo for encouragements and his interests in this work, helpful discussions and comments; to Z. Chang, B. Feng, E. Gava, H. -Y. Guo, K. S. Narain, K. Wu, Y. -S. Wu, Z. Xu and Z. -X. Zhang for discussions and comments; to Prof. X. -Q. Li and the hospitality at Nankai University where we can have good food; and finally to Prof. S. Randjbar-Daemi and the hospitality at Abdus Salam International Center for Theoretical Physics, Trieste, Italy. This work is supported in part by funds from the National Natural Science Foundation of China with grant number 10475104 and 10525522.

\section{References}

[1] Z. -G. Xiao, G. Yang and C. -J. Zhu, "The rational parts of one-loop QCD amplitudes I: The general formalism," hep-ph/0607015.

[2] X. Su, Z. -G. Xiao, G. Yang and C. -J. Zhu, "The rational parts of one-loop QCD amplitudes II: The 5-gluon case," hep-ph/0607016. 
[3] Z. Bern, L. J. Dixon and D. A. Kosower, "One Loop Corrections to Five Gluon Amplitudes," Phys. Rev. Lett. 70 (1993) 2677-2680, hep$\mathrm{ph} / 9302280$.

[4] Z. Bern, L. Dixon and D. A. Kosower, "New QCD Results from String Theory," talk presented by Z. Bern at Strings 1993, May 24-29, Berkeley CA, USA, hep-th/9311026; Z. Bern, G. Chalmers, L. Dixon and D. A. Kosower, "One-Loop N Gluon Amplitudes with Maximal Helicity Violation via Collinear Limits," Phys. Rev. Lett. 72 (1994) 2134-2137, hep-ph/9312333.

[5] G. Mahlon, "Multigluon Helicity Amplitudes Involving a Quark Loop," Phys. Rev. D49 (1994) 4438-4453, hep-ph/9312276.

[6] Z. Bern, L. J. Dixon and D. A. Kosower, "On-Shell Recurrence Relations for One-Loop QCD Amplitudes," Phys. Rev. D71 (2005) 105013, hep-th/0501240; "The Last of the Finite Loop Amplitudes in QCD," Phys. Rev. D72 (2005) 125003, hep-ph/0505055.

[7] Z. Bern, L. J. Dixon and D. A. Kosower, "Bootstrapping Multi-Parton Loop Amplitudes in QCD," Phys. Rev. D73 (2006) 065013, hep$\mathrm{ph} / 0507005$.

[8] C. F. Berger, Z. Bern, L. J. Dixon, D. Forde and D. A. Kosower, "Bootstrapping One-Loop QCD Amplitudes with General Helicities," hep-ph/0604195.

[9] C. F. Berger, Z. Bern, L. J. Dixon, D. Forde and D. A. Kosower, "All One-loop Maximally Helicity Violating Gluonic Amplitudes in QCD," hep-ph/0607014.

[10] G. P. Salam, "Developments in perturbative QCD," Invited talk at 22nd International Symposium on Lepton-Photon Interactions at High Energy (LP 2005), Uppsala, Sweden, 30 Jun - 5 Jul 2005, hep$\mathrm{ph} / 0510090$.

[11] E. Witten, "Perturbative gauge theory as a string theory in twistor space," Commun. Math. Phys. 252 (2004) 189-258, hep-th/0312171. 
[12] F. Cachazo, P. Svrček and E. Witten, "MHV vertices and tree amplitudes in gauge theory," JHEP 0409, (2004) 006, hep-th/0403047.

[13] R. K. Ellis and J, C. Sexton, "QCD Radiative Corrections to PartonParton Scattering," Nucl. Phys. B269 (1986) 445-484.

[14] Z. Bern and D. A. Kosower, "Efficient Computation Of Gauge Theory Loops Using String Theory," in Copenhagen 1987, Proceedings, Perspectives In String Theory, p. 390-402; "A New Approach To One Loop Calculations In Gauge Theories," Phys. Rev. D38 (1988) 1888; "Efficient calculation of one loop QCD amplitudes," Phys. Rev. Lett. 66 (1991) 1669-1672; "The Computation of loop amplitudes in gauge theories," Nucl. Phys. B379 (1992) 451-561.

[15] Z. Bern, L. Dixon, D. C. Dunbar and D. A. Kosower, "One-Loop n-Point Gauge Theory Amplitudes, Unitarity and Collinear Limits," Nucl. Phys. B425 (1994) 217-260, hep-ph/9403226; "Fusing Gauge Theory Tree Amplitudes Into Loop Amplitudes," Nucl. Phys. B435 (1995) 59-101, hep-ph/9409265.

[16] Z. Bern, "String based perturbative methods for gauge theories," TASI 92 lectures, hep-ph/9304249.

[17] Z. Xu, D.-H. Zhang and L. Chang, "Helicity Amplitudes For Multiple Bremsstrahlung In Massless Nonabelian Theories," Nucl. Phys. B291 (1987) 392.

[18] L. J. Dixon, "Calculating Scattering Amplitudes Efficiently," hep$\mathrm{ph} / 9601359$.

[19] Z. Kunszt, A. Signer and Z. Trócsányi, "One-loop radiative corrections to the helicity amplitudes of QCD processes involving four quarks and one gluon," Phys. Lett. B336 (1994) 529-536, hep-ph/9405386.

[20] Z. Bern, L. J. Dixon and D. A. Kosower, "One-loop corrections to two-quark three-gluon amplitudes," Nucl. Phys. B437 (1995) 259-304, hep-ph/9409393. 
[21] T. Binoth, J. P. Guillet, G. Heinrich and C. Schubert, "Calculation of 1-loop Hexagon Amplitudes in the Yukawa Model," Nucl. Phys. B615 (2001)

[22] A. Brandhuber, B. Spence and G. Travaglini, "One-Loop Gauge Theory Amplitudes In $\mathrm{N}=4$ Super Yang-Mills From MHV Vertices," Nucl. Phys. B 706 (2005) 150-180, hep-th/0407214; "From Trees to Loops and Back," JHEP 0601 (2006) 142, hep-th/0510253.

[23] F. Cachazo, "Holomorphic Anomaly Of Unitarity Cuts And One-Loop Gauge Theory Amplitudes," hep-th/0410077.

[24] Z. Bern, V. Del Duca, L. J. Dixon and D. A. Kosower, "All NonMaximally-Helicity-Violating One-Loop Seven-Gluon Amplitudes in N=4 Super-Yang-Mills Theory," Phys. Rev. D71 (2005) 045006, hepth/0410224;

S. J. Bidder, N. E. J. Bjerrum-Bohr, L, J. Dixon and D. C. Dunbar, "N=1 Supersymmetric One-loop Amplitudes and the Holomorphic Anomaly of Unitarity Cuts," Phys. Lett. B606 (2005) 189-201, hep-th/0410296;

Z. Bern, L. J. Dixon and D. A. Kosower, "All Next-to-MaximallyHelicity-Violating One-Loop Gluon Amplitudes in N=4 Super-YangMills Theory," Phys. Rev. D72 (2005) 045014, hep-th/0412210.

[25] C. Quigley and M. Rozali, "One-Loop MHV Amplitudes in Supersymmetric Gauge Theories," JHEP 0501 (2005) 053, hep-th/0410278.

[26] J. Bedford, A. Brandhuber, B. Spence and G. Travaglini, "A Twistor Approach to One-Loop Amplitudes in $\mathrm{N}=1$ Supersymmetric YangMills Theory," Nucl. Phys. B706 (2005) 100-126, hep-th/0410280; "Non-Supersymmetric Loop Amplitudes and MHV Vertices," Nucl. Phys. B712 (2005) 59-85, hep-th/0412108.

[27] S. J. Bidder, N.E.J. Bjerrum-Bohr, D. C. Dunbar and W. B. Perkins, "Twistor Space Structure of the Box Coefficients of N=1 One-loop Amplitudes," Phys. Lett. B608 (2005) 151-163, hep-th/0412023; 
S. J. Bidder, N. E. J. Bjerrum-Bohr, D. C. Dunbar and W. B. Perkins, "One-Loop Gluon Scattering Amplitudes in Theories with $N<4$ Supersymmetries," Phys. Lett. B612 (2005) 75-88, hep-th/0502028.

[28] Z. Bern, N. E. J. Bjerrum-Bohr, D. C. Dunbar and H. Ita, "Recursive Calculation of One-Loop QCD Integral Coefficients," JHEP 0511 (2005) 027, hep-ph/0507019.

[29] M. -X. Luo and C. -K. Wen, "One-Loop Maximal Helicity Violating Amplitudes in N=4 Super Yang-Mills Theories," JHEP 0411 (2004) 004, hep-th/0410045; "Systematics of One-Loop Scattering Amplitudes in N=4 Super Yang-Mills Theories," Phys. Lett. B609 (2005) 86-94, hep-th/0410118.

[30] R. Britto, F. Cachazo and B. Feng, "New Recursion Relations for Tree Amplitudes of Gluons," Nucl. Phys. B715 (2005) 499-522, hepth/0412308;

R. Britto, F. Cachazo, B. Feng and E. Witten, "Direct Proof Of TreeLevel Recursion Relation In Yang-Mills Theory," Phys. Rev. Lett. 94 (2005) 181602, hep-th/0501052.

[31] R. Britto, F. Cachazo and B. Feng, "Generalized Unitarity and OneLoop Amplitudes in N=4 Super-Yang-Mills," Nucl.Phys. B725 (2005) 275-305, hep-th/0412103.

[32] R. Britto, E. Buchbinder, F. Cachazo, B. Feng, "One-Loop Amplitudes Of Gluons In SQCD," Phys. Rev. D72 (2005) 065012, hep-ph/0503132.

[33] R. Britto, B. Feng and P. Mastrolia, "The Cut-Constructible Part of QCD Amplitudes," Phys. Rev. D73 (2006) 105004, hep-ph/0602178.

[34] Z. Bern, L. Dixon and D. A. Kosower, "One-loop amplitudes for $e^{+} e^{-}$ to four partons," Nucl. Phys. B513 (1998) 3-86, hep-ph/9708239.

[35] Z. Bern and A. G. Morgan, "Massive Loop Amplitudes from Unitarity," Nucl. Phys. B467 (1996) 479-509, hep-ph/9511336.

[36] For reviews see: Z. Bern, L. Dixon and D. A. Kosower, "Unitaritybased Techniques for One-Loop Calculations in QCD," Nucl. Phys. 
Proc. Suppl. 51C (1996) 243-249, hep-ph/9606378; "Progress in OneLoop QCD Computations," Ann. Rev. Nucl. Part. Sci. 46 (1996) 109148, hep-ph/9602280.

[37] A. Brandhuber, S. McNamara, B. Spence and G. Travaglini, "Loop Amplitudes in Pure Yang-Mills from Generalised Unitarity," JHEP 0510 (2005) 011, hep-th/0506068.

[38] D. Forde and D. A. Kosower, "All-Multiplicity One-Loop Corrections to MHV Amplitudes in QCD," Phys. Rev. D73 (2006) 061701, hepph/0509358.

[39] A. Berends and W. T. Giele, "Recursive Calculations For Processes With N Gluons," Nucl. Phys. B306 (1988) 759.

[40] G. Passarino and M. J. G. Veltman, "One-loop Corrections for $e^{+} e^{-}$ annihilation into $\mu^{+} \mu^{-}$in the Weinberg modes," Nucl. Phys. B160 (1979) 151-207.

[41] D. B. Melrose, "Reduction Of Feynman Diagrams," Nuovo Cim. 40, 181 (1965); W. L. van Neerven and J. A. M. Vermaseren, "Large Loop Integrals," Phys. Lett. B 137, 241 (1984).

[42] Z. Bern, L. Dixon and D. A. Kosower, "Dimensionally Regulated OneLoop Integrals," Phys.Lett. B302 (1993) 299-308, Erratum-ibid. B318 (1993) 649, hep-ph/9212308; "Dimensionally Regulated Pentagon Integrals," Nucl. Phys. B412 (1994) 751-816, hep-ph/9306240.

[43] T. Binoth, J.Ph. Guillet, G. Heinrich, "Reduction formalism for dimensionally regulated one-loop N-point integrals," Nucl.Phys. B572 (2000) 361-386, hep-ph/9911342.

[44] O. V. Tarasov, "Connection between Feynman integrals having different values of the space-time dimension," Phys. Rev. D 54, 6479 (1996), hep-th/9606018; J. Fleischer, F. Jegerlehner and O. V. Tarasov, "Algebraic reduction of one-loop Feynman graph amplitudes," Nucl. Phys. B 566, 423 (2000), hep-ph/9907327. 
[45] G. Duplancic and B. Nizic, "Reduction method for dimensionally regulated one-loop N-point Feynman integrals," Eur. Phys. J. C 35, 105 (2004), hep-ph/0303184.

[46] A. Denner and S. Dittmaier, "Reduction of one-loop tensor 5-point integrals," Nucl. Phys. B 658, 175 (2003), hep-ph/0212259; "Reduction schemes for one-loop tensor integrals," Nucl. Phys. B 734, 62 (2006), hep-ph/0509141 and the references therein.

[47] Z. Bern and G. Chalmers, "Factorization in one-loop gauge theory," Nucl. Phys. B447 (1995) 465-518, hep-th/9503236. 